\title{
TOEPLITZ AND HANKEL OPERATORS ON BERGMAN SPACES
}

\author{
KAREL STROETHOFF AND DECHAO ZHENG
}

\begin{abstract}
In this paper we consider Toeplitz and Hankel operators on the Bergman spaces of the unit ball and the polydisk in $\mathbb{C}^{n}$ whose symbols are bounded measurable functions. We give necessary and sufficient conditions on the symbols for these operators to be compact. We study the Fredholm theory of Toeplitz operators for which the corresponding Hankel operator is compact. For these Toeplitz operators the essential spectrum is computed and shown to be connected. We also consider symbols that extend to continuous functions on the maximal ideal space of $H^{\infty}(\Omega)$; for these symbols we describe when the Toeplitz or Hankel operators are compact.
\end{abstract}

\section{INTRODUCTION}

For a bounded domain $\Omega$ in $\mathbb{C}^{n}$, let $V$ denote the Lebesgue measure on $\Omega$ normalized so that $\Omega$ has measure 1 . For $1 \leq p<\infty$ and a Lebesgue measurable function $f: \Omega \rightarrow \mathbb{C}$ let $\|f\|_{p}=\left(\int_{\Omega}|f(z)|^{p} d V(z)\right)^{1 / p}$. The Bergman space $L_{a}^{p}(\Omega)$ is the Banach space of analytic functions $f: \Omega \rightarrow \mathbb{C}$ such that $\|f\|_{p}<\infty$. The Bergman space $L_{a}^{2}(\Omega)$ is a closed subspace of the Hilbert space $L^{2}(\Omega, d V)$ with inner product given by $\langle f, g\rangle=\int_{\Omega} f(z) \overline{g(z)} d V(z)$, for $f, g \in L^{2}(\Omega, d V)$. Let $P$ denote the orthogonal projection of $L^{2}(\Omega, d V)$ onto $L_{a}^{2}(\Omega)$. For a function $f \in L^{\infty}(\Omega)$, the Toeplitz operator $T_{f}: L_{a}^{2}(\Omega) \rightarrow$ $L_{a}^{2}(\Omega)$ and the Hankel operator $H_{f}: L_{a}^{2}(\Omega) \rightarrow L_{a}^{2}(\Omega)^{\perp}$ are defined by

$$
T_{f} g=P(f g), \quad H_{f} g=(I-P)(f g), \quad g \in L_{a}^{2}(\Omega) .
$$

These are clearly bounded operators for every function $f \in L^{\infty}(\Omega)$. In this paper we consider the question of characterizing the functions $f \in L^{\infty}(\Omega)$ for which these operators are compact on $L_{a}^{2}(\Omega)$, for $\Omega$ the open unit ball $\mathbb{B}_{n}=\left\{z \in \mathbb{C}^{n}:|| z||<1\right\}$ or the polydisk $\mathbb{D}^{n}=\left\{z \in \mathbb{C}^{n}:\left|z_{j}\right|<1\right.$ for $\left.1 \leq j \leq n\right\}$ in $\mathbb{C}^{n}$. The question of characterizing the bounded measurable functions on $\mathbb{D}$, the unit disk in $\mathbb{C}$, for which the Hankel operator $H_{f}$ is compact was raised by Sheldon Axler in [1]. Sheldon Axler answered a special case of this problem in [2] where he considered conjugate analytic symbols on the unit disk $\mathbb{D}$. Recently both of the authors independently obtained a complete answer for

Received by the editors December 11, 1989.

1980 Mathematics Subject Classification (1985 Revision). Primary 47B35, 47B38.

The first author was partially supported by grants from the Montana University System and the University of Montana. 
Axler's question in $[17,18,21]$. In this paper we will combine and extend our results.

Throughout the paper let $\Omega$ denote either the unit ball $\mathbb{B}_{n}$ or the polydisk $\mathbb{D}^{n}$. In our characterization of the compact Toeplitz and Hankel operators on $L_{a}^{2}(\Omega)$ the Möbius transformations on $\Omega$ will play a crucial role: for each $\lambda \in \Omega$ there is an automorphism of $\Omega$ with the following properties:

(i) $\varphi_{\lambda}(\lambda)=0$, and

(ii) $\varphi_{\lambda} \circ \varphi_{\lambda}=\mathrm{id}_{\Omega}$ (the identity map on $\Omega$ ).

For the unit ball $\mathbb{B}_{n}$ these Möbius transformations are described in $\S 2.2$ of [16]; for the polydisk $\mathbb{D}^{n}$ they are described in $\S 10.1$ of [13].

We denote the topological boundary of $\Omega$ in $\mathbb{C}^{n}$ by $\partial \Omega$. The statement $\lambda \rightarrow \partial \Omega$ will simply mean that $\lambda \in \Omega$ and the usual distance of $\lambda$ to $\partial \Omega$ tends to 0 .

In the first part of the paper $(\S \S 2-4)$ we consider general bounded symbols on $\Omega$. In $\S 2$ we will give the preliminaries needed for the rest of the paper. In $\S 3$ we will give our characterization of the compact Toeplitz and Hankel operators. Our main result in this section states that for $\Omega$ the unit ball or the polydisk in $\mathbb{C}^{n}$ the Toeplitz operator $T_{f}$ is a compact operator on $L_{a}^{2}(\Omega)$ if and only if for some $p \in[1, \infty),\left\|P\left(f \circ \varphi_{\lambda}\right)\right\|_{p} \rightarrow 0$ as $\lambda \rightarrow \partial \Omega$; and the Hankel operator $H_{f}$ is a compact operator on $L_{a}^{2}(\Omega)$ if and only if for some $p \in[1, \infty),\left\|f \circ \varphi_{\lambda}-P\left(f \circ \varphi_{\lambda}\right)\right\|_{p} \rightarrow 0$ as $\lambda \rightarrow \partial \Omega$. We give several descriptions for compactness of Hankel operators with Berezin symbols in $\S 4$. In $\S 5$ we will consider Toeplitz operators of bounded symbols for which the Hankel operator is compact. For these Toeplitz operators we describe the essential spectrum, give necessary and sufficient conditions for the operator to be Fredholm, and for the Toeplitz operators which are Fredholm we prove an index theorem. In $\S 6$ we specialize to symbols that extend to continuous functions on the maximal ideal space of $H^{\infty}(\Omega)$. For these symbols we give a condition for compactness of the Toeplitz operator, and we give several conditions equivalent to compactness of the Hankel operator. We end the paper with some remarks and open questions in $\S 7$.

\section{Preliminaries}

Point evaluation is a bounded linear functional on the Hilbert space $L_{a}^{2}(\Omega)$, thus for every $\lambda \in \Omega$ there exists a unique analytic function $k_{\lambda} \in L_{a}^{2}(\Omega)$ such that

$$
f(\lambda)=\left\langle f, k_{\lambda}\right\rangle, \quad \text { for all } f \in L_{a}^{2}(\Omega) .
$$

These functions $k_{\lambda}(\lambda \in \Omega)$ are called the reproducing kernels for $L_{a}^{2}(\Omega)$. For the unit ball and the polydisk in $\mathbb{C}^{n}$ these reproducing kernels can be computed explicitly (see, for example, $\S 1.4$ in [13]): for $\Omega=\mathbb{B}_{n}$ we have $k_{\lambda}(z)=(1-\langle z, \lambda\rangle)^{-n-1}\left(z \in \mathbb{B}_{n}\right)$. For $f \in L^{\infty}(\Omega), g \in L^{2}(\Omega, d V)$ and $z \in \Omega$ we have $\left(T_{f} g\right)(z)=\left\langle P(f g), k_{z}\right\rangle=\left\langle f g, k_{z}\right\rangle$, so we get the following formula for $T_{f} g$ :

$$
\left(T_{f} g\right)(z)=\int_{\Omega} f(w) g(w) \overline{k_{z}(w)} d V(w), \quad z \in \Omega .
$$

Also using the reproducing property of $k_{z}$ we get the following formula for 
$H_{f} g:$

$$
\left(H_{f} g\right)(z)=\int_{\Omega}(f(z)-f(w)) g(w) \overline{k_{z}(w)} d V(w), \quad z \in \Omega .
$$

For $\lambda \in \Omega$ the substitution $z=\varphi_{\lambda}(w)$ results in the Jacobian change in measure given by $d V(w)=\left(J_{\mathbb{R}} \varphi_{\lambda}\right)(z) d V(z)$, where $J_{\mathbb{R}} \varphi_{\lambda}$ is the real Jacobian of $\varphi_{\lambda}$. Using that $J_{\mathbb{R}} \varphi_{\lambda}=\left|J_{\mathbb{C}} \varphi_{\lambda}\right|^{2}$ (Proposition 1.4.10 in [13]), it is easy to verify that $J_{\mathbb{R}} \varphi_{\lambda}$ is given by the formula $\left(J_{\mathbb{R}} \varphi_{\lambda}\right)(z)=\left|k_{\lambda}(z)\right|^{2} / k_{\lambda}(\lambda)$. Thus, for a Lebesgue integrable or nonnegative Lebesgue measurable function $h$ on $\Omega$ we have the change-of-variable formula:

$$
\int_{\Omega} h\left(\varphi_{\lambda}(w)\right) d V(w)=\frac{1}{k_{\lambda}(\lambda)} \int_{\Omega} h(z)\left|k_{\lambda}(z)\right|^{2} d V(z) .
$$

In the next section we will give our characterization of compact Toeplitz and Hankel operators. The following proposition, which gives formulas for the images of the reproducing kernels $k_{\lambda}(\lambda \in \Omega)$ under the operators $T_{f}$ and $H_{f}$, will play an important role in our characterization.

Proposition 1. Let $f \in L^{\infty}(\Omega)$. For each $\lambda \in \Omega$ we have

$$
T_{f}\left(k_{\lambda}\right)=\left(P\left(f \circ \varphi_{\lambda}\right) \circ \varphi_{\lambda}\right) k_{\lambda}
$$

and

$$
H_{f}\left(k_{\lambda}\right)=\left(f-P\left(f \circ \varphi_{\lambda}\right) \circ \varphi_{\lambda}\right) k_{\lambda} .
$$

Proof. Take $f \in L^{\infty}(\Omega)$ and $\lambda \in \Omega$. Clearly it suffices to prove (4). Let $z \in \Omega$. Using (3) we see that for a function $g \in L_{a}^{2}(\Omega)$ we have $\left\langle\left(f \circ \varphi_{\lambda}\right) k_{\lambda},\left(g \circ \varphi_{\lambda}\right) k_{\lambda}\right\rangle=$ $k_{\lambda}(\lambda)\langle f, g\rangle=k_{\lambda}(\lambda)\langle P(f), g\rangle=\left\langle\left(P(f) \circ \varphi_{\lambda}\right) k_{\lambda},\left(g \circ \varphi_{\lambda}\right) k_{\lambda}\right\rangle$. Replacing $f$ by $f \circ \varphi_{\lambda}$ and $g$ by $\left(g \circ \varphi_{\lambda}\right) /\left(k_{\lambda} \circ \varphi_{\lambda}\right)$ (which using that the $k_{\lambda}$ never vanish and are bounded on $\Omega$ is easily seen to belong to $L_{a}^{2}(\Omega)$ ) we see that for every $g \in L_{a}^{2}(\Omega)$ we have $\left\langle\left(P\left(f \circ \varphi_{\lambda}\right) \circ \varphi_{\lambda}\right) k_{\lambda}, g\right\rangle=\left\langle f k_{\lambda}, g\right\rangle$, from which we conclude that $T_{f}\left(k_{\lambda}\right)=P\left(f k_{\lambda}\right)=\left(P\left(f \circ \varphi_{\lambda}\right) \circ \varphi_{\lambda}\right) k_{\lambda}$.

\section{COMPACt ToEplitz AND HANKel OPERATORS}

In this section we will characterize the functions $f$ in $L^{\infty}(\Omega)$ for which the Toeplitz operator $T_{f}$ or the Hankel operator $H_{f}$ is compact. We will need estimates on certain integrals involving the reproducing kernels. For $q \in[1, \infty)$ and $\varepsilon>0$ we introduce the notation

$$
M_{q, \varepsilon}(\Omega)=\sup _{\lambda \in \Omega} \int_{\Omega}\left|k_{\lambda}(w)\right|^{(1-2 \varepsilon) q} k_{w}(w)^{\varepsilon q} d V(w) .
$$

It will be important to know when these quantities are finite. The following lemma tells us when this is the case for $\Omega$ the unit ball or the polydisk in $\mathbb{C}^{n}$.

Lemma 2. Let $\Omega$ be the unit ball or the polydisk in $\mathbb{C}^{n}$. Then there exist $q_{\Omega}>1$ and $\varepsilon_{\Omega}>0$ such that $M_{q, \varepsilon}(\Omega)<\infty$ for every $q \in\left[1, q_{\Omega}\right)$ and $\varepsilon \in\left(0, \varepsilon_{\Omega}\right]$.

Proof. For $\Omega=\mathbb{B}_{n}$ it is an easy consequence of Proposition 1.4.10 in [16] that the above statement holds with $q_{\Omega}=(2 n+2) /(2 n+1)$ and $\varepsilon_{\Omega}=$ $1 /(2 n+2)$. Using that the reproducing kernel of the polydisk is the product of the reproducing kernels of the disk, the statement for $\Omega=\mathbb{D}^{n}$ follows 
from that for the disk $\mathbb{D}=\mathbb{B}_{1}:$ the numbers $q_{\Omega}=4 / 3$ and $\varepsilon_{\Omega}=1 / 4$ are easily seen to give the desired statement.

The following lemma gives an estimate that will be used in the proofs of Theorems 6 and 7, our characterization of compact Toeplitz and Hankel operators on the Bergman space $L_{a}^{2}(\Omega)$.

Lemma 3. Let $F$ be a nonnegative measurable function on $\Omega \times \Omega, 1<q<\infty$, $p=q /(q-1)$ and $\varepsilon>0$. Then for every $w \in \Omega$ :

$$
\begin{aligned}
& \int_{\Omega} F\left(w, \varphi_{w}(z)\right)\left|k_{w}(z)\right| k_{z}(z)^{\varepsilon} d V(z) \\
& \quad \leq k_{w}(w)^{\varepsilon} M_{q, \varepsilon}(\Omega)^{1 / q}\left(\int_{\Omega} F(w, z)^{p} d V(z)\right)^{1 / p} .
\end{aligned}
$$

Proof. Let $F$ be a nonnegative measurable function on $\Omega \times \Omega$. In the integral at the left make the change-of-variable $z=\varphi_{w}(u)$. Using (3) we get

$$
\begin{aligned}
\int_{\Omega} F\left(w, \varphi_{w}(z)\right)\left|k_{w}(z)\right| k_{z}(z)^{\varepsilon} d V(z) \\
=k_{w}(w)^{\varepsilon} \int_{\Omega} F(w, u)\left|k_{w}(u)\right|^{1-2 \varepsilon} k_{u}(u)^{\varepsilon} d V(u) \\
\leq k_{w}(w)^{\varepsilon} M_{q, \varepsilon}(\Omega)^{1 / q}\left(\int_{\Omega} F(w, z)^{p} d V(z)\right)^{1 / p}
\end{aligned}
$$

(by Hölder's inequality).

In the proofs of Theorems 6 and 7 we will also need the estimates contained in the following lemma.

Lemma 4. Let $f \in L^{\infty}(\Omega)$ and $\varepsilon>0$. Then for every $z \in \Omega$ :

$$
\int_{\Omega}\left|P\left(f \circ \varphi_{w}\right)\left(\varphi_{w}(z)\right)\right|\left|k_{w}(z)\right| k_{w}(w)^{\varepsilon} d V(w) \leq M_{1, \varepsilon}(\Omega)^{2}\|f\|_{\infty} k_{z}(z)^{\varepsilon}
$$

and

$$
\begin{aligned}
\int_{\Omega} \mid f(z) & -P\left(f \circ \varphi_{w}\right)\left(\varphi_{w}(z)\right)|| k_{w}(z) \mid k_{w}(w)^{\varepsilon} d V(w) \\
\leq & 2 M_{1, \varepsilon}(\Omega)^{2}\|f\|_{\infty} k_{z}(z)^{\varepsilon} .
\end{aligned}
$$

Proof. Let $f \in L^{\infty}(\Omega), \varepsilon>0$, and $z \in \Omega$. We will only show (6); the proof of (7) will be similar. Using (4) and (1) we have

$$
\begin{aligned}
\left|P\left(f \circ \varphi_{w}\right)\left(\varphi_{w}(z)\right)\right|\left|k_{w}(z)\right| & =\left|\left(T_{f} k_{w}\right)(z)\right|=\left|\int_{\Omega} f(u) k_{w}(u) \overline{k_{z}(u)} d V(u)\right| \\
& \leq\|f\|_{\infty} \int_{\Omega}\left|k_{w}(u)\right|\left|k_{z}(u)\right| d V(u) .
\end{aligned}
$$

Thus

$$
\begin{aligned}
\int_{\Omega} \mid P\left(f \circ \varphi_{w}\right) & \left(\varphi_{w}(z)\right)|| k_{w}(z) \mid k_{w}(w)^{\varepsilon} d V(w) \\
& \leq\|f\|_{\infty} \int_{\Omega}\left|k_{z}(u)\right|\left(\int_{\Omega}\left|k_{w}(u)\right| k_{w}(w)^{\varepsilon} d V(w)\right) d V(u)
\end{aligned}
$$


As in the proof of Lemma 2, a change-of-variable yields

$$
\int_{\Omega}\left|k_{w}(u)\right| k_{w}(w)^{\varepsilon} d V(w) \leq M_{1, \varepsilon}(\Omega) k_{u}(u)^{\varepsilon} .
$$

Applying inequality (9) twice in (8) we get (6).

To estimate the norms of certain (integral) operators we will make use of the Schur Test as stated in the following proposition [11, Theorem 5.2].

Proposition 5. Let $(X, \nu)$ be a measure space and $K$ a measurable function on $X \times X$. Suppose there is a positive measurable function $h$ on $X$ and positive numbers $\alpha$ and $\beta$ such that

$$
\int_{X}|K(x, y)| h(y) d \nu(y) \leq \alpha h(x) \text { for }[\nu]-\text { a.e. } x \text { in } X
$$

and

$$
\int_{X}|K(x, y)| h(x) d \nu(x) \leq \beta h(y) \quad \text { for }[\nu]-\text { a.e. } y \text { in } X \text {. }
$$

Then

$$
(A f)(x)=\int_{X} K(x, y) f(y) d \nu(y), \quad f \in L^{2}(X, \nu) \text { and } x \in X,
$$

defines a bounded linear operator $A$ from $L^{2}(X, \nu)$ into itself. Moreover, $\|A\|^{2} \leq \alpha \beta$.

We are now ready to state and prove our main result, contained in Theorems 6 and 7. The proofs of these theorems will be combined into one proof.

Theorem 6. Let $f \in L^{\infty}(\Omega)$. The following statements are equivalent:

(a) $T_{f}$ is compact;

(b) $\left\|P\left(f \circ \varphi_{\lambda}\right)\right\|_{p} \rightarrow 0$ as $\lambda \rightarrow \partial \Omega$ for some $p \in[1, \infty)$;

(c) $\left\|P\left(f \circ \varphi_{\lambda}\right)\right\|_{p} \rightarrow 0$ as $\lambda \rightarrow \partial \Omega$ for every $p \in[1, \infty)$.

Theorem 7. Let $f \in L^{\infty}(\Omega)$. The following statements are equivalent:

(a) $H_{f}$ is compact;

(b) $\left\|f \circ \varphi_{\lambda}-P\left(f \circ \varphi_{\lambda}\right)\right\|_{p} \rightarrow 0$ as $\lambda \rightarrow \partial \Omega$ for some $p \in[1, \infty)$;

(c) $\left\|f \circ \varphi_{\lambda}-P\left(f \circ \varphi_{\lambda}\right)\right\|_{p} \rightarrow 0$ as $\lambda \rightarrow \partial \Gamma$ for every $p \in[1, \infty)$.

Proof of Theorems 6 and 7. Fix a function $f \in L^{\infty}(\Omega)$. Let $M_{f}$ be the multiplication operator $L_{a}^{2}(\Omega) \rightarrow L^{2}(\Omega, d V)$ defined by $M_{f}(g)=f g$ for $g \in L_{a}^{2}(\Omega)$. Writing $Q$ for either $P$ or $I-P$ we note that both $H_{f}$ and $T_{f}$ are of the form $Q M_{f}$; the proofs of Theorems 6 and 7 will be combined into one proof.

Proof that $(\mathrm{a}) \Rightarrow(\mathrm{b})$. Suppose that the operator $Q M_{f}$ is compact. By the reproducing property we have that $\left\|k_{\lambda}\right\|_{2}^{2}=k_{\lambda}(\lambda)$. If $g$ is a bounded analytic function on $\Omega$, then $\left\langle g, k_{\lambda} /\left\|k_{\lambda}\right\|_{2}\right\rangle=k_{\lambda}(\lambda)^{-1 / 2} g(\lambda) \rightarrow 0$ as $\lambda \rightarrow \partial \Omega$ (because $k_{\lambda}(\lambda) \rightarrow \infty$ as $\left.\lambda \rightarrow \partial \Omega\right)$. Since the bounded analytic functions on $\Omega$ are dense in the Bergman space $L_{a}^{2}(\Omega)$, this shows that $k_{\lambda} /\left\|k_{\lambda}\right\|_{2} \rightarrow 0$ weakly in $L_{a}^{2}(\Omega)$ as $\lambda \rightarrow \partial \Omega$. Compact operators map weakly null sequences to norm null sequences, thus we have $\left\|Q M_{f}\left(k_{\lambda} /\left\|k_{\lambda}\right\|_{2}\right)\right\|_{2} \rightarrow 0$ as $\lambda \rightarrow \partial \Omega$. By Proposition $1, Q M_{f}\left(k_{\lambda}\right)=\left(Q\left(f \circ \varphi_{\lambda}\right) \circ \varphi_{\lambda}\right) k_{\lambda}$. Using change-of-variable formula (3) it is 
easily seen that $\left\|Q M_{f}\left(k_{\lambda} /\left\|k_{\lambda}\right\|_{2}\right)\right\|_{2}=\left\|Q\left(f \circ \varphi_{\lambda}\right)\right\|_{2}$ thus $\left\|Q\left(f \circ \varphi_{\lambda}\right)\right\|_{2} \rightarrow 0$ as $\lambda \rightarrow \partial \Omega$.

Proof that $(\mathrm{b}) \Rightarrow(\mathrm{c})$. Suppose that $\left\|Q\left(f \circ \varphi_{\lambda}\right)\right\|_{p_{0}} \rightarrow 0$ as $\lambda \rightarrow \partial \Omega$ for some $p_{0} \in[1, \infty)$. Let $p \in[1, \infty)$ be arbitrary. If $p \leq p_{0}$, then $\left\|Q\left(f \circ \varphi_{\lambda}\right)\right\|_{p} \leq$ $\left\|Q\left(f \circ \varphi_{\lambda}\right)\right\|_{p_{0}}$, thus $\left\|Q\left(f \circ \varphi_{\lambda}\right)\right\|_{p} \rightarrow 0$ as $\lambda \rightarrow \partial \Omega$. If $p>p_{0}$, then using Hölder's inequality it is easy to verify that

$$
\left\|Q\left(f \circ \varphi_{\lambda}\right)\right\|_{p}^{p} \leq\left\|Q\left(f \circ \varphi_{\lambda}\right)\right\|_{p_{0}}^{1 / 2}\left\|Q\left(f \circ \varphi_{\lambda}\right)\right\|_{q}^{p-1 / 2},
$$

where $q=p_{0}(2 p-1) /\left(2 p_{0}-1\right)>1$. Now, since the Bergman projection $P$ maps $L^{q}(\Omega, d V)$ boundedly onto $L_{a}^{q}(\Omega)$ and $Q$ is either $P$ or $I-P$, there is a constant $C_{q}$ such that

$$
\left\|Q\left(f \circ \varphi_{\lambda}\right)\right\|_{q} \leq C_{q}\left\|Q\left(f \circ \varphi_{\lambda}\right)\right\|_{q} \leq C_{q}\|f\|_{\infty} .
$$

Hence $\left\|Q\left(f \circ \varphi_{\lambda}\right)\right\|_{p}^{p} \leq\left(C_{q}\|f\|_{\infty}\right)^{p-1 / 2}\left\|Q\left(f \circ \varphi_{\lambda}\right)\right\|_{p_{0}}^{1 / 2}$, which immediately implies that also $\left\|Q\left(f \circ \varphi_{\lambda}\right)\right\|_{p} \rightarrow 0$ as $\lambda \rightarrow \partial \Omega$.

Proof that (c) $\Rightarrow(\mathrm{a})$. Suppose that $\left\|Q\left(f \circ \varphi_{\lambda}\right)\right\|_{p} \rightarrow 0$ as $\lambda \rightarrow \partial \Omega$ for every $p \in[1, \infty)$. We will prove that the operator $\left(Q M_{f}\right)^{*}$ is compact by showing that $\left(Q M_{f}\right)^{*}$ can be approximated-in the operator norm-by compact operators. have

Let $g \in Q\left(L^{2}(\Omega, d V)\right)$. Then $\left(Q M_{f}\right)^{*} g \in L_{a}^{2}(\Omega)$, so that for $w \in \Omega$ we

$$
\begin{aligned}
\left(\left(Q M_{f}\right)^{*} g\right)(w) & =\left\langle\left(Q M_{f}\right)^{*} g, k_{w}\right\rangle=\left\langle g,\left(Q\left(f \circ \varphi_{w}\right) \circ \varphi_{w}\right) k_{w}\right\rangle \\
& =\int_{\Omega} g(z) \overline{Q\left(f \circ \varphi_{w}\right)\left(\varphi_{w}(z)\right) k_{w}(z)} d V(z) .
\end{aligned}
$$

In view of this we define for $0<r<1$ an operator $S_{r}: Q\left(L^{2}(\Omega, d V)\right) \rightarrow L_{a}^{2}(\Omega)$ by

$$
\left(S_{r} g\right)(w)=\chi_{r \Omega}(w) \int_{\Omega} g(z) \overline{Q\left(f \circ \varphi_{w}\right)\left(\varphi_{w}(z)\right) k_{w}(z)} d V(z),
$$

for $g \in Q\left(L^{2}(\Omega, d V)\right), w \in \Omega$. Then $S_{r}$ is a Hilbert-Schmidt operator. To see this, observe that change-of-variable formula (3) and Fubini's Theorem give

$$
\begin{gathered}
\int_{\Omega}\left(\int_{\Omega} \chi_{r \Omega}(w)\left|Q\left(f \circ \varphi_{w}\right)\left(\varphi_{w}(z)\right)\right|^{2}\left|k_{w}(z)\right|^{2} d V(z)\right) d V(z) \\
=\int_{r \Omega} k_{w}(w)\left\|Q\left(f \circ \varphi_{w}\right)\right\|_{2}^{2} d V(w)<\infty,
\end{gathered}
$$

and it follows that $S_{r}$ is Hilbert-Schmidt. Using (12) and the definition of $S_{r}$ we see that for $h \in Q\left(L^{2}(\Omega, d V)\right)$ and $w \in \Omega$ :

$$
\left(\left(\left(Q M_{f}\right)^{*}-S_{r}\right) g\right)(w)=\int_{\Omega} K(w, z) g(z) d V(z)
$$

where $K(w, z)=\chi_{\Omega \backslash r \Omega}(w) \overline{Q\left(f \circ \varphi_{w}\right)\left(\varphi_{w}(z)\right) k_{w}(z)}$. We claim that $S_{r} \rightarrow$ $\left(Q M_{f}\right)^{*}$ in operator norm as $r \rightarrow 1$. Since the $S_{r}$ are compact, it will follow from this claim that $\left(Q M_{f}\right)^{*}$ is compact, and therefore $Q M_{f}$ is compact. To prove our claim we will apply Proposition 5 to get an estimate on the operator norm $\left\|\left(Q M_{f}\right)^{*}-S_{r}\right\|$. For $\varepsilon=\varepsilon_{\Omega}$, the constant from Lemma 2, let $h(w)=$ $k_{w}(w)^{\varepsilon}(w \in \Omega)$. By Lemma 4 inequality (11) in Proposition 5 is satisfied with $\beta=2 M_{1, \varepsilon}(\Omega)^{2}\|f\|_{\infty}$. For $w, z \in \Omega$ put $F(w, z)=\chi_{\Omega \backslash\ulcorner\Omega}(w)\left|Q\left(f \circ \varphi_{w}\right)(z)\right|$. 
Let $q_{\Omega}$ be as in Lemma 2. Choose $q \in\left(1, q_{\Omega}\right)$, and let $p=q /(q-1)$ be the conjugate exponent of $q$. Using that $|K(w, z)|=F\left(w, \varphi_{w}(z)\right)\left|k_{w}(z)\right|$, Lemma 3 tells us

$$
\begin{aligned}
\int_{\Omega} K(w, z) k_{z}(z)^{\varepsilon} d V(z) & \leq k_{w}(w)^{\varepsilon} M_{q, \varepsilon}(\Omega)^{1 / q}\left(\int_{\Omega} F(w, z)^{p} d V(z)\right)^{1 / p} \\
& =k_{w}(w)^{\varepsilon} M_{q, \varepsilon}(\Omega)^{1 / q} \chi_{\Omega \backslash r \Omega}(w)\left\|Q\left(f \circ \varphi_{w}\right)\right\|_{p},
\end{aligned}
$$

so that inequality (10) in Proposition 5 holds with

$$
\alpha=M_{q, \varepsilon}(\Omega)^{1 / q} \sup \left\{\left\|Q\left(f \circ \varphi_{\lambda}\right)\right\|_{p}: \lambda \in \Omega \backslash r \Omega\right\} .
$$

It follows from Proposition 5 that

$$
\left\|\left(Q M_{f}\right)^{*}-S_{r}\right\|^{2} \leq 2 M_{1, \varepsilon}(\Omega)^{2} M_{q, \varepsilon}(\Omega)^{1 / q}\|f\|_{\infty} \sup _{\lambda \in \Omega \backslash r \Omega}\left\|Q\left(f \circ \varphi_{\lambda}\right)\right\|_{p} .
$$

Since $\left\|Q\left(f \circ \varphi_{\lambda}\right)\right\|_{p} \rightarrow 0$ as $\lambda \rightarrow \partial \Omega$ our claim that $S_{r} \rightarrow\left(Q M_{f}\right)^{*}$ in operator norm as $r \rightarrow 1^{-}$follows.

For $f \in L^{2}(\Omega, d V)$ define $\tilde{f}$, the Berezin symbol of $f$, by

$$
\begin{aligned}
\tilde{f}(\lambda) & =\left\langle f k_{\lambda} /\left\|k_{\lambda}\right\|_{2}, k_{\lambda} /\left\|k_{\lambda}\right\|_{2}\right\rangle \\
& =\frac{1}{k_{\lambda}(\lambda)} \int_{\Omega} f(z)\left|k_{\lambda}(z)\right|^{2} d V(z), \quad \lambda \in \Omega .
\end{aligned}
$$

Note that by change-of-variable formula (3), $\tilde{f}(\lambda)=\int_{\Omega} f \circ \varphi_{\lambda} d V(\lambda \in \Omega)$. Thus for a function $f \in L_{a}^{2}(\Omega)$ we have, $\int_{\Omega} f \circ \varphi_{\lambda} d V=\left(f \circ \varphi_{\lambda}\right)(0)=f(\lambda)$, and therefore $\widetilde{f}=f$.

As in the above proof, for $f \in L^{\infty}(\Omega)$ let $M_{f}$ be the multiplication operator $L_{a}^{2}(\Omega) \rightarrow L^{2}(\Omega, d V)$ defined by $M_{f}(g)=f g$ for $g \in L_{a}^{2}(\Omega)$. As an easy corollary of Theorems 6 and 7 we get the following result.

Corollary 8. Let $f \in L^{\infty}(\Omega)$. The following statements are equivalent:

(a) $M_{f}$ is compact;

(b) $\mid \widetilde{\left.f\right|^{2}}(\lambda) \rightarrow 0$ as $\lambda \rightarrow \partial \Omega$.

Proof. It is easily verified that for $\lambda \in \Omega$ :

$$
\mid \widetilde{\left.f\right|^{2}}(\lambda)=\left\|f \circ \varphi_{\lambda}\right\|_{2}^{2}=\left\|P\left(f \circ \varphi_{\lambda}\right)\right\|_{2}^{2}+\left\|f \circ \varphi_{\lambda}-P\left(f \circ \varphi_{\lambda}\right)\right\|_{2}^{2}
$$

So if $\mid \widetilde{\left.f\right|^{2}}(\lambda) \rightarrow 0$ as $\lambda \rightarrow \partial \Omega$, then both

$$
\left\|P\left(f \circ \varphi_{\lambda}\right)\right\|_{2} \rightarrow 0 \text { and }\left\|f \circ \varphi_{\lambda}-P\left(f \circ \varphi_{\lambda}\right)\right\|_{2} \rightarrow 0 \quad \text { as } \lambda \rightarrow \partial \Omega,
$$

so that by Theorems 6 and 7 both $T_{f}$ and $H_{f}$ are compact, thus $M_{f}$ is compact.

It is easily seen that $\mid \widetilde{\left.f\right|^{2}}(\lambda)=\left\|M_{f}\left(k_{\lambda} /\left\|k_{\lambda}\right\|_{2}\right)\right\|_{2}^{2}$. We have already observed that $k_{\lambda} /\left\|k_{\lambda}\right\|_{2} \rightarrow 0$ weakly in $L_{a}^{2}(\Omega)$ as $\lambda \rightarrow \partial \Omega$, so if $M_{f}$ is compact, then $\mid \widetilde{\left.f\right|^{2}}(\lambda) \rightarrow 0$ as $\lambda \rightarrow \partial \Omega$.

In the next section we will frequently make use of the following consequence of the above corollary. 
Corollary 9. Let $f \in L^{\infty}(\Omega)$ be supported on a compact subset of $\Omega$. Then both $T_{f}$ and $H_{f}$ are compact.

Proof. If $f \in L^{\infty}(\Omega)$ has compact support, then $\mid \widetilde{\left.f\right|^{2}}(\lambda) \rightarrow 0$ as $\lambda \rightarrow \partial \Omega$.

As a corollary of Theorem 7 we also obtain some of the main results in [8].

Corollary 10. Let $f \in L^{\infty}(\Omega)$. The following statements are equivalent:

(a) $H_{f}$ and $H_{\bar{f}}$ are compact;

(b) $\left\|f \circ \varphi_{\lambda}-\widetilde{f}(\lambda)\right\|_{p} \rightarrow 0$ as $\lambda \rightarrow \partial \Omega$ for some $p \in(1, \infty)$;

(c) $\left\|f \circ \varphi_{\lambda}-\widetilde{f}(\lambda)\right\|_{p} \rightarrow 0$ as $\lambda \rightarrow \partial \Omega$ for every $p \in(1, \infty)$.

Proof. Proof that $(\mathrm{a}) \Rightarrow(\mathrm{c})$. Suppose that $H_{f}$ and $H_{\bar{f}}$ are compact and $p \in(1, \infty)$. Then

$$
\left\|f \circ \varphi_{\lambda}-\overline{P\left(\bar{f} \circ \varphi_{\lambda}\right)}\right\|_{p}=\left\|\bar{f} \circ \varphi_{\lambda}-P\left(\bar{f} \circ \varphi_{\lambda}\right)\right\|_{p} \rightarrow 0 \quad \text { as } \lambda \rightarrow \partial \Omega .
$$

By continuity of $P$ we get $\left\|P\left(f \circ \varphi_{\lambda}\right)-\tilde{f}(\lambda)\right\|_{p}=\left\|P\left(f \circ \varphi_{\lambda}-\overline{P\left(\bar{f} \circ \varphi_{\lambda}\right)}\right)\right\|_{p} \rightarrow 0$ as $\lambda \rightarrow \partial \Omega$. Combining this with $\left\|f \circ \varphi_{\lambda}-P\left(f \circ \varphi_{\lambda}\right)\right\|_{p} \rightarrow 0$ as $\lambda \rightarrow \partial \Omega$ statement (c) follows.

That $(c) \Rightarrow(b)$ is trivial.

Proof that $(\mathrm{b}) \Rightarrow(\mathrm{a})$. Suppose that $\left\|f \circ \varphi_{\lambda}-\tilde{f}(\lambda)\right\|_{p} \rightarrow 0$ as $\lambda \rightarrow \partial \Omega$ for some $p \in(1, \infty)$. Again using the continuity of $P$ it follows that

$$
\left\|P\left(f \circ \varphi_{\lambda}\right)-\tilde{f}(\lambda)\right\|_{p} \rightarrow 0 \quad \text { as } \lambda \rightarrow \partial \Omega,
$$

thus $\left\|f \circ \varphi_{\lambda}-P\left(f \circ \varphi_{\lambda}\right)\right\|_{p} \rightarrow 0$ as $\lambda \rightarrow \partial \Omega$. By Theorem $7, H_{f}$ is compact. Since also $\left\|\bar{f} \circ \varphi_{\lambda}-\tilde{\bar{f}}(\lambda)\right\|_{p} \rightarrow 0$ as $\lambda \rightarrow \partial \Omega$ the above reasoning shows that $H_{\bar{f}}$ is also compact.

In $\S 5$ we will discuss the essential spectrum of the Toeplitz operators for which the corresponding Hankel operator is compact. We will need some results relating the Toeplitz and Hankel operator associated to $\widetilde{f}$ with those associated to $f$. This will be done in Proposition 12. First we need a lemma.

Lemma 11. Let $g \in L^{2}(\Omega, d V)$ and $\varepsilon>0$. Then $\|\tilde{g}\|_{2} \leq 2^{n+1} M_{1, \varepsilon}(\Omega)\|g\|_{2}$. Proof. It is readily verified that $\left|k_{w}(z)\right| \leq 2^{n+1} k_{w}(w)$ (remember that $\Omega$ is the unit ball or the polydisk in $\left.\mathbb{C}^{n}\right)$, so that for $g \in L^{2}(\Omega, d V)$ and $w \in \Omega$

$$
|\widetilde{g}(w)| \leq 2^{n+1} \int_{\Omega}|g(z)|\left|k_{w}(z)\right| d V(z) .
$$

Using (9) and Proposition 5 (with $\left.h(w)=k_{w}(w)^{\varepsilon}\right)$ the statement follows easily.

Proposition 12. Let $f \in L^{\infty}(\Omega)$ be such that $H_{f}$ is compact. Then:

(1) $T_{f-\tilde{f}}$ is compact;

(2) $H_{\tilde{f}}$ is compact.

Proof. Suppose $f \in L^{\infty}(\Omega)$ is such that $H_{f}$ is compact. Let $\varepsilon=\varepsilon_{\Omega}$ be as in Lemma 2. Let $\lambda \in \Omega$. Apply Lemma 11 with $g=f \circ \varphi_{\lambda}-P\left(f \circ \varphi_{\lambda}\right)$. Since $P\left(f \circ \varphi_{\lambda}\right) \in L_{a}^{2}(\Omega)$ its Berezin symbol is equal to itself. Also, if $w \in \Omega$, then by Cartan's Theorem [16, Theorem 2.1.3] there exists a unitary $n \times n$ matrix $U$ 
such that $U(\Omega)=\Omega$ and $\varphi_{\lambda} \circ \varphi_{w}=\varphi_{\varphi_{\lambda}(w)} \circ U$, and consequently $\left(f \circ \varphi_{\lambda}\right)^{\sim}(w)=$ $\int_{\Omega} f \circ \varphi_{\lambda} \circ \varphi_{w} d V=\int_{\Omega} f \circ \varphi_{\varphi_{\lambda}(w)} d V=\widetilde{f}\left(\varphi_{\lambda}(w)\right)$. Thus $\tilde{g}=\tilde{f} \circ \varphi_{\lambda}-P\left(f \circ \varphi_{\lambda}\right)$, and by Lemmas 2 and 11 there is a firite constant $C$ such that for every $\lambda \in \Omega$ :

$$
\left\|\tilde{f} \circ \varphi_{\lambda}-P\left(f \circ \varphi_{\lambda}\right)\right\|_{2} \leq C\left\|f \circ \varphi_{\lambda}-P\left(f \circ \varphi_{\lambda}\right)\right\|_{2} \text {. }
$$

By Theorem 7 we have $\left\|f \circ \varphi_{\lambda}-P\left(f \circ \varphi_{\lambda}\right)\right\|_{2} \rightarrow 0$ as $\lambda \rightarrow \partial \Omega$, so that by (14) $\left\|\widetilde{f} \circ \varphi_{\lambda}-P\left(f \circ \varphi_{\lambda}\right)\right\|_{2} \rightarrow 0$ as $\lambda \rightarrow \partial \Omega$, hence $\left\|f \circ \varphi_{\lambda}-\widetilde{f} \circ \varphi_{\lambda}\right\|_{2} \rightarrow 0$ as $\lambda \rightarrow \partial \Omega$. By continuity of $P,\left\|P\left(\tilde{f} \circ \varphi_{\lambda}-f \circ \varphi_{\lambda}\right)\right\|_{2} \rightarrow 0$ as $\lambda \rightarrow \partial \Omega$, and by Theorem 6 it follows that $T_{f-\tilde{f}}$ is compact. Also $\left\|\widetilde{f} \circ \varphi_{\lambda}-P\left(\tilde{f} \circ \varphi_{\lambda}\right)\right\|_{2} \rightarrow 0$ as $\lambda \rightarrow \partial \Omega$, so that also $H_{\widetilde{f}}$ is compact (by Theorem 7).

\section{HANKEL OPERATORS WITH BEREZIN SYMBOLS}

In this section we will give several descriptions for compactness of a Hankel operator associated with the Berezin symbol of a bounded measurable function.

It is easy to see that the Berezin symbol of a bounded measurable function is continuous. We will write $B C(\Omega)$ to denote the algebra of bounded continuous functions on $\Omega$. Let $\beta \Omega$ denote the Stone-Čech compactification of $\Omega$. Every function $f$ in $B C(\Omega)$ has a unique continuous extension to $\beta \Omega$ which we will denote by $f^{\beta}$. Let $\Phi$ be the set of all possible limits in the product space $(\beta \Omega)^{\Omega}$ of nets $\left\{\varphi_{\lambda_{\alpha}}\right\}$ for which $\lambda_{\alpha} \rightarrow \partial \Omega$. Note that by Tychonoff's Theorem the space $(\beta \Omega)^{\Omega}$ is compact, so that every net $\left\{\lambda_{\alpha}\right\}$ in $\Omega$ tending to $\partial \Omega$ has a subnet $\left\{w_{\gamma}\right\}$ such that $\left\{\varphi_{w_{\gamma}}\right\}$ converges in $(\beta \Omega)^{\Omega}$ to some $\varphi \in \Phi$. Also note that if $\left\{\varphi_{\alpha}\right\}$ is a net in $(\beta \Omega)^{\Omega}$ such that $\varphi_{\alpha} \rightarrow \varphi$ in $(\beta \Omega)^{\Omega}$, then for all $\lambda \in \Omega$, $\varphi_{\alpha}(\lambda) \rightarrow \varphi(\lambda)$ in $\beta \Omega$ (because the projection of $(\beta \Omega)^{\Omega}$ onto its $\lambda$-coordinate is continuous). So if also $f \in B C(\Omega)$, then $f \circ \varphi_{\alpha} \rightarrow f^{\beta} \circ \varphi$ pointwise on $\Omega$. The $\Phi$-parts of $\beta \Omega$ are by definition the images of the elements in $\Phi$. We introduce two subalgebras of $B C(\Omega)$. Let $A O \Phi$ (which stands for "analytic on $\Phi$-parts") be defined by

$$
A O \Phi=\left\{f \in B C(\Omega): f^{\beta} \circ \varphi \in H^{\infty}(\Omega) \text { for all } \varphi \in \Phi\right\},
$$

and let $C O \Phi$ (which stands for "constant on $\Phi$-parts") be defined by

$$
C O \Phi=\left\{f \in B C(\Omega): f^{\beta} \circ \varphi \text { is constant for all } \varphi \in \Phi\right\} .
$$

In the following theorem we will relate these algebras to compactness of Hankel operators with Berezin symbols. We first need to introduce the Bergman metric and recall a few facts about this metric proved in [6].

The Bergman metric $b$ is a complete Riemannian metric on $\Omega$ which gives the usual topology on $\Omega$. By definition, $b$ is the integrated form of the infinitesimal metric

$$
\left(g_{j m}(z)\right)=\left(\frac{1}{2} \frac{\partial^{2}}{\partial z_{j} \partial \bar{z}_{m}} \log k_{z}(z)\right) .
$$

As a special case of Theorem $F$ in [6] we have that for a smooth curve $\sigma:[0,1]$ $\rightarrow \Omega$ and a $f \in L^{\infty}(\Omega)$

$$
\left|\frac{d}{d t} \tilde{f}(\sigma(t))\right| \leq C_{f} \frac{d s}{d t}
$$


for some positive constant $C_{f}$ only depending on $f$, where $s=s(t)$ is the arclength of $\sigma$ with respect to the Bergman metric $b$. Choosing for $\sigma$ a geodesic joining two points $z$ and $w$ in $\Omega$ it follows immediately that

$$
|\widetilde{f}(z)-\tilde{f}(w)| \leq C_{f} b(z, w), \quad \text { for } z, w \in \Omega .
$$

We have already noted that if $g \in B C(\Omega)$ and $\left\{w_{\alpha}\right\}$ is a net in $\Omega$ tending to $\partial \Omega$ such that $\varphi_{w_{\alpha}} \rightarrow \varphi$ in $(\beta \Omega)^{\Omega}$, then $g \circ \varphi_{w_{\alpha}} \rightarrow g^{\beta} \circ \varphi$ pointwise on $\Omega$. The following lemma shows that for Berezin symbols this convergence is uniform on compact subsets of $\Omega$.

Lemma 13. Let $f \in L^{\infty}(\Omega)$, and let $\left\{w_{\alpha}\right\}$ be a net in $\Omega$ tending to $\partial \Omega$ such that $\varphi_{w_{\alpha}} \rightarrow \varphi$ in $(\beta \Omega)^{\Omega}$. Then $\tilde{f} \circ \varphi_{w_{\alpha}}$ converges to $\tilde{f}^{\beta} \circ \varphi$ uniformly on each compact subset of $\Omega$.

Proof. Suppose that $f \in L^{\infty}(\Omega)$. We have already seen that $\tilde{f} \circ \varphi_{w_{\alpha}}$ converges to $\tilde{f}^{\beta} \circ \varphi$ pointwise on $\Omega$, so it suffices to prove that the family $\left\{\tilde{f} \circ \varphi_{w_{\alpha}}\right\}$ is equicontinuous on each compact subset of $\Omega$. Inequality (16) and the Möbiusinvariance of the Bergman metric $b$ imply that $\left|\left(\tilde{f} \circ \varphi_{w_{\alpha}}\right)(z)-\left(\tilde{f} \circ \varphi_{w_{\alpha}}\right)(w)\right| \leq$ $C_{f} b(z, w)$, from which the equicontinuity of the family $\left\{\tilde{f} \circ \varphi_{w_{\alpha}}\right\}$ follows at once.

The following theorem relates the algebras $A O \Phi$ and $C O \Phi$ to compactness of the Hankel operator associated with the Berezin symbol of a bounded measurable function.

Theorem 14. Let $f \in L^{\infty}(\Omega)$. Then:

(1) $H_{\tilde{f}}$ is compact if and only if $\widetilde{f} \in A O \Phi$;

(2) both $H_{\widetilde{f}}$ and $H_{\overline{\tilde{f}}}$ are compact if and only if $\tilde{f} \in C O \Phi$.

Proof. Since clearly $C O \Phi=A O \Phi \cap \overline{A O \Phi}$ it suffices to prove (1). By Theorem 7 we have that $H_{\widetilde{f}}$ is compact if and only if $\left\|\widetilde{f} \circ \varphi_{\lambda}-P\left(\tilde{f} \circ \varphi_{\lambda}\right)\right\|_{2} \rightarrow 0$ as $\lambda \rightarrow \partial \Omega$. By the previous lemma, if $\left\{w_{\alpha}\right\}$ is a net in $\Omega$ tending to $\partial \Omega$ such that $\varphi_{w_{\alpha}} \rightarrow \varphi$ in $(\beta \Omega)^{\Omega}$, then $\left\|\widetilde{f} \circ \varphi_{w_{\alpha}}-\widetilde{f}^{\beta} \circ \varphi\right\|_{2} \rightarrow 0$. Using this observation, the compactness of $H_{\widetilde{f}}$ is easily seen to be equivalent with $\left\|\tilde{f}^{\beta} \circ \varphi-P\left(\tilde{f}^{\beta} \circ \varphi\right)\right\|_{2}=0$, i.e., $\tilde{f}^{\beta} \circ \varphi=P\left(\tilde{f}^{\beta} \circ \varphi\right)$, for every $\varphi \in \Omega$.

In the sequel we will also need the following lemma.

Lemma 15. Let $\varphi \in \Phi$ and $w \in \Omega$. Then there exist $a \psi \in \Phi$ and a unitary $n$ by $n$ matrix $U$ such that $U(\Omega)=\Omega$ and $\varphi \circ \varphi_{w}=\psi \circ U$.

Proof. Fix $\varphi \in \Phi$ and $w \in \Omega$, and let $\left\{w_{\alpha}\right\}$ be a net in $\Omega$ such that $w_{\alpha} \rightarrow \partial \Omega$ and $\varphi_{w_{\alpha}} \rightarrow \varphi$ in $(\beta \Omega)^{\Omega}$. By Cartan's Theorem [16, Theorem 2.1.3] there is a unitary $n$ by $n$ matrix $U_{\alpha}$ such that $U_{\alpha}(\Omega)=\Omega$ and $\varphi_{w_{\alpha}} \circ \varphi_{w}=\varphi_{z_{\alpha}} \circ U_{\alpha}$, where $z_{\alpha}=\varphi_{w_{\alpha}}(w)$. It is easy to verify that also $z_{\alpha} \rightarrow \partial \Omega$. Using that the unitary group is compact, we may assume-by going to a subnet which we do not relabel-that $\left\{\varphi_{z_{\alpha}}\right\}$ converges in $(\beta \Omega)^{\Omega}$, say to $\psi$, and that $\left\{U_{\alpha}\right\}$ converges in the unitary group, say to $U$. Clearly $U(\Omega)=\Omega$, and since $z_{\alpha} \rightarrow \partial \Omega$, $\psi \in \Phi$. It is now readily verified that $\varphi \circ \varphi_{w}=\psi \circ U$.

For $w \in \Omega$ and $r \in(0,1)$ let the pseudohyperbolic ball $\Omega(w, r)$, with center $w$ and radius $r$, be defined by $\Omega(w, r)=\varphi_{w}(r \Omega)$. For the unit ball 
$\mathbb{B}_{n}$ these pseudohyperbolic balls are ellipsoids (see $\$ 2.2 .7$ in [16]); for the polydisk $\mathbb{D}^{n}$ these pseudohyperbolic balls are cartesian products of pseudohyperbolic disks in $\mathbb{D}$.

The following proposition gives a more precise description of the algebra $A O \Phi$. The result is a generalization of Sheldon Axler's description of $A O P$ for the unit disk [3]. The "distance" in the statement of this proposition is with respect to the supremum norm.

Proposition 16. Let $f \in L^{\infty}(\Omega)$. Then the following statements are equivalent:

(i) $\tilde{f} \in A O \Phi$;

(ii) $\operatorname{distance}\left(\tilde{f} \mid \Omega(w, r), H^{\infty}(\Omega(w, r))\right) \rightarrow 0$ as $w \rightarrow \partial \Omega$ for every $r \in$ $(0,1)$;

(iii) distance $\left(\tilde{f} \mid \Omega(w, r), H^{\infty}(\Omega(w, r))\right) \rightarrow 0$ as $w \rightarrow \partial \Omega$ for some $r \in$ $(0,1)$.

Proof. Let $f \in L^{\infty}(\Omega)$.

Proof that (i) $\Rightarrow$ (ii). Suppose that (ii) does not hold. Then there exist $r \in(0,1), \delta>0$, and a sequence $\left\{w_{j}\right\}$ in $\Omega$ such that $w_{j} \rightarrow \partial \Omega$ as $j \rightarrow \infty$, and

$$
\operatorname{distance}\left(\tilde{f} \mid \Omega\left(w_{j}, r\right), H^{\infty}\left(\Omega\left(w_{j}, r\right)\right)\right) \geq \delta, \quad \text { for all } j \geq 1 \text {. }
$$

Let $\left\{w_{\alpha}\right\}$ be a subnet of $\left\{w_{j}\right\}$ such that $\left\{\varphi_{w_{\alpha}}\right\}$ converges in $(\beta \Omega)^{\Omega}$, say to $\varphi \in \Phi$. Then by Lemma $13, \tilde{f} \circ \varphi_{w_{\alpha}} \rightarrow \tilde{f}^{\beta} \circ \varphi$ uniformly on $r \Omega$. For every index $\alpha$ we have

$$
\operatorname{distance}\left(\tilde{f} \circ \varphi_{w_{\alpha}} \mid r \Omega, H^{\infty}(r \Omega)\right) \geq \delta,
$$

so it follows that $\operatorname{distance}\left(\tilde{f}^{\beta} \circ \varphi \mid r \Omega, H^{\infty}(r \Omega)\right) \geq \delta$, which implies that $\tilde{f}^{\beta} \circ \varphi \notin$ $H^{\infty}(\Omega)$, thus $\tilde{f} \notin A O \Phi$. This proves that (i) implies (ii).

That (ii) $\Rightarrow$ (iii) is trivial.

Proof that (iii) $\Rightarrow(\mathrm{i})$. Suppose that $r \in(0,1)$ is such that

$$
\operatorname{distance}\left(\tilde{f} \mid \Omega(w, r), H^{\infty}(\Omega(w, r))\right) \rightarrow 0 \quad \text { as } w \rightarrow \partial \Omega \text {. }
$$

Take $\varphi \in \Phi$. Let $\left\{w_{\alpha}\right\}$ be a net in $\Omega$ such that $w_{\alpha} \rightarrow \partial \Omega$ and $\varphi_{w_{\alpha}} \rightarrow \varphi$ in $(\beta \Omega)^{\Omega}$. For each index $\alpha$ let $g_{\alpha} \in H^{\infty}\left(\Omega\left(w_{\alpha}, r\right)\right)$ be such that

$$
\left\|\tilde{f} \mid \Omega\left(w_{\alpha}, r\right)-g_{\alpha}\right\|_{\infty} \leq 2 \operatorname{distance}\left(\tilde{f} \mid \Omega\left(w_{\alpha}, r\right), H^{\infty}\left(\Omega\left(w_{\alpha}, r\right)\right)\right) .
$$

Then $\left\|\tilde{f} \mid \Omega\left(w_{\alpha}, r\right)-g_{\alpha}\right\|_{\infty} \rightarrow 0$. Putting $h_{\alpha}=g_{\alpha} \circ \varphi_{w_{\alpha}}$, we have

$$
\left\|\tilde{f} \circ \varphi_{w_{\alpha}} \mid r \Omega-h_{\alpha}\right\|_{\infty} \rightarrow 0 .
$$

Since $\left\{\tilde{f} \circ \varphi_{w_{\alpha}}\right\}$ is uniformly bounded on $r \Omega$, there is no loss of generality in assuming that the same is true for $\left\{h_{\alpha}\right\}$. Then there is a subnet $\left\{h_{\nu}\right\}$ and $h \in H^{\infty}(r \Omega)$ such that $h_{\nu} \rightarrow h$ weak-star in $H^{\infty}(r \Omega)$. In particular, $h_{\nu} \rightarrow h$ pointwise on $r \Omega$. But also $\tilde{f} \circ \varphi_{w_{\nu}} \rightarrow \tilde{f}^{\beta} \circ \varphi$ pointwise on $r \Omega$, and $\| \tilde{f} \circ$ $\varphi_{w_{\nu}} \mid r \Omega-h_{\nu} \|_{\infty} \rightarrow 0$. We conclude that $\tilde{f}^{\beta} \circ \varphi=h$ on $r \Omega$, thus $\widetilde{f}^{\beta} \circ \varphi$ is analytic on $r \Omega$.

Now let $w \in \Omega$ be arbitrary. By Lemma 15 there is a unitary $n$ by $n$ matrix $U$ and a $\psi \in \Phi$ such that $U(\Omega)=\Omega$ and $\varphi \circ \varphi_{w}=\psi \circ U$. By the previous paragraph $\tilde{f}^{\beta} \circ \psi$ is analytic on $r \Omega$. Because $U$ maps $r \Omega$ analytically onto 
itself, we see that $\tilde{f}^{\beta} \circ \psi \circ U$ is analytic on $r \Omega$, i.e., $\tilde{f}^{\beta} \circ \varphi \circ \varphi_{w}$ is analytic on $r \Omega$. Thus $\tilde{f}^{\beta} \circ \varphi$ is analytic on $\Omega(w, r)$. Because $w \in \Omega$ was an arbitrary point of $\Omega, \widetilde{f}^{\beta} \circ \varphi$ is analytic on all of $\Omega$. This holds for every $\varphi \in \Phi$, hence $\widetilde{f} \in A O \Phi$.

Remark. It is clear from the proof that the above proposition remains true if the pseudohyperbolic balls $\Omega(w, r)$ are replaced by sets $\varphi_{w}(G)$, where $G$ is a fixed nonempty open subset of $\Omega$ whose closure is contained in $\Omega$. In particular, the proposition holds for the balls with respect to the Bergman metric.

The following proposition gives a description of the algebra $C O \Phi$ in terms of the oscillations over pseudohyperbolic balls of a fixed (pseudohyperbolic) radius. The result is a generalization of Sheldon Axler's description of COP for the unit disk [3].

Proposition 17. Let $f \in L^{\infty}(\Omega)$. Then the following statements are equivalent:

(i) $\tilde{f} \in C O \Phi$;

(ii) $\sup \{|\tilde{f}(z)-\tilde{f}(w)|: z \in \Omega(w, r)\} \rightarrow 0$ as $w \rightarrow \partial \Omega$ for every $r \in(0,1)$;

(iii) $\sup \{|\tilde{f}(z)-\tilde{f}(w)|: z \in \Omega(w, r)\} \rightarrow 0$ as $w \rightarrow \partial \Omega$ for some $r \in(0,1)$. Proof. Let $f \in L^{\infty}(\Omega)$.

Proof that (i) $\Rightarrow$ (ii). Suppose that (ii) does not hold. Then there exist $r \in(0,1), \delta>0$, and sequences $\left\{w_{j}\right\}$ in $\Omega$ and $\left\{\lambda_{j}\right\}$ in $r \Omega$ such that $w_{j} \rightarrow \partial \Omega$ as $j \rightarrow \infty$ and

$$
\left|\left(\tilde{f} \circ \varphi_{w_{j}}\right)\left(\lambda_{j}\right)-\tilde{f}\left(w_{j}\right)\right| \geq \delta, \quad \text { for all } j \geq 1 .
$$

Let $\left\{w_{\alpha}\right\}$ be a subnet of $\left\{w_{j}\right\}$ such that $\varphi_{w_{\alpha}} \rightarrow \varphi$ in $(\beta \Omega)^{\Omega}$ for some $\varphi \in \Phi$. Since the net $\left\{\lambda_{\alpha}\right\}$ is contained in a compact subset of $\Omega$, by going to a subnet (that we will not relabel) we may assume that $\lambda_{\alpha} \rightarrow \lambda$ for some $\lambda \in \Omega$. By Lemma 13 we have $\left(\tilde{f} \circ \varphi_{w_{\alpha}}\right)\left(\lambda_{\alpha}\right) \rightarrow\left(\tilde{f}^{\beta} \circ \varphi\right)(\lambda)$. On the other hand, $\tilde{f}\left(w_{\alpha}\right)=$ $\left(\tilde{f} \circ \varphi_{w_{\alpha}}\right)(0) \rightarrow\left(\tilde{f}^{\beta} \circ \varphi\right)(0)$. Since for every index $\alpha,\left|\left(\tilde{f} \circ \varphi_{w_{\alpha}}\right)\left(\lambda_{\alpha}\right)-\tilde{f}\left(w_{\alpha}\right)\right| \geq \delta$, it follows that $\left|\left(\tilde{f}^{\beta} \circ \varphi\right)(\lambda)-\left(\tilde{f}^{\beta} \circ \varphi\right)(0)\right| \geq \delta$, hence $\tilde{f}^{\beta} \circ \varphi$ is not constant. This proves that (i) implies (ii).

That (ii) $\Rightarrow$ (iii) is trivial.

Proof that (iii) $\Rightarrow$ (i). Suppose that $\sup \{|\widetilde{f}(z)-\widetilde{f}(w)|: z \in \Omega(w, r)\} \rightarrow 0$ as $w \rightarrow \partial \Omega$ for some $r \in(0,1)$. Take $\varphi \in \Phi$. Let $\left\{w_{\alpha}\right\}$ be a net in $\Omega$ such that $w_{\alpha} \rightarrow \partial \Omega$ and $\varphi_{w_{\alpha}} \rightarrow \varphi$ in $(\beta \Omega)^{\Omega}$. For $\lambda \in r \Omega, \mid\left(\tilde{f} \circ \varphi_{w_{\alpha}}\right)(\lambda)-$ $\tilde{f}\left(w_{\alpha}\right) \mid \leq \sup \left\{\left|\tilde{f}(z)-\tilde{f}\left(w_{\alpha}\right)\right|: z \in \Omega\left(w_{\alpha}, r\right)\right\}$, thus $\left(\tilde{f} \circ \varphi_{w_{\alpha}}\right)(\lambda)-\tilde{f}\left(w_{\alpha}\right) \rightarrow 0$. On the other hand, $\left(\tilde{f} \circ \varphi_{w_{\alpha}}\right)(\lambda) \rightarrow\left(\tilde{f}^{\beta} \circ \varphi\right)(\lambda)$ and $\tilde{f}\left(w_{\alpha}\right) \rightarrow\left(\tilde{f}^{\beta} \circ \varphi\right)(0)$. Thus $\left(\tilde{f}^{\beta} \circ \varphi\right)(\lambda)=\left(\tilde{f}^{\beta} \circ \varphi\right)(0)$, and we conclude that $\tilde{f}^{\beta} \circ \varphi$ is constant on $r \Omega$.

Now let $w \in \Omega$ be arbitrary. By Lemma 15 there is a unitary $n$ by $n$ matrix $U$ and a $\psi \in \Phi$ such that $U(\Omega)=\Omega$ and $\varphi \circ \varphi_{w}=\psi \circ U$. By the previous paragraph $\tilde{f}^{\beta} \circ \psi$ is constant on $r \Omega$, so $\tilde{f}^{\beta} \circ \psi \circ U$ is constant on $r \Omega$, i.e., $\widetilde{f}^{\beta} \circ \varphi \circ \varphi_{w}$ is constant on $r \Omega$. Thus $\tilde{f}^{\beta} \circ \varphi$ is constant on $\Omega(w, r)$. By an easy covering argument we conclude that $\tilde{f}^{\beta} \circ \varphi$ is constant on all of $\Omega$. This holds for every $\varphi \in \Phi$, hence $\widetilde{f} \in C O \Phi$.

Remark. Again it is clear from the proof that the above proposition is also true if the pseudohyperbolic balls $\Omega(w, r)$ are replaced by sets $\varphi_{w}(G)$, where 
$G$ is a fixed nonempty open subset of $\Omega$ whose closure is contained in $\Omega$. In particular, the proposition holds for the balls with respect to the Bergman metric.

\section{The ESSENTIAL SPECTRUM OF TOEPLITZ OPERATORS}

Let $\mathscr{L}\left(L_{a}^{2}(\Omega)\right)$ denote the Banach algebra of the bounded linear operators on $L_{a}^{2}(\Omega)$, and let $\mathscr{K}$ denote the ideal of compact operators in $\mathscr{L}\left(L_{a}^{2}(\Omega)\right.$ ). For an operator $T$ in $\mathscr{L}\left(L_{a}^{2}(\Omega)\right)$ the essential spectrum of $T$, denoted by $\sigma_{e}(T)$, is by definition the spectrum of the operator $T+\mathscr{K}$ in the Calkin algebra $\mathscr{L}\left(L_{a}^{2}(\Omega)\right) / \mathscr{K}$, i.e., $\sigma_{e}(T)$ is the set of all complex numbers $\zeta$ such that $T-\zeta+\mathscr{K}$ is not invertible in $\mathscr{L}\left(L_{a}^{2}(\Omega)\right) / \mathscr{K}$.

Let $A Q(\Omega)=\left\{f \in L^{\infty}(\Omega): H_{f}\right.$ is compact $\}$. It is well known that $A Q(\Omega)$ is a closed subalgebra of $L^{\infty}(\Omega)$ (see for example p. 475 of [4]). In this section we will describe the essential spectrum of the Toeplitz operator $T_{f}$ for $f$ in the algebra $A Q(\Omega)$.

For $E \subset \mathbb{C}^{n}$, let cl $E$ denote the closure of the set $E$ in $\mathbb{C}^{n}$. It is easy to verify that $C\left(\operatorname{cl} \mathbb{B}_{n}\right) \subset A Q\left(\mathbb{B}_{n}\right)$. Since trivially $H^{\infty}\left(\mathbb{B}_{n}\right) \subset A Q\left(\mathbb{B}_{n}\right)$, it follows that the algebra $H^{\infty}\left(\mathbb{B}_{n}\right)+C\left(\mathrm{cl} \mathbb{B}_{n}\right)$ is contained in $A Q\left(\mathbb{B}_{n}\right)$. Thus the results in this section generalize the results of [14].

We will need the following lemma.

Lemma 18. Let $F \in A Q(\Omega)$ be such that for some $\delta>0,|F(w)| \geq \delta$ for all $w \in \Omega$. Then $1 / F \in A Q(\Omega)$.

Proof. Suppose $F \in A Q(\Omega)$ is such that for some $\delta>0,|F(w)| \geq \delta$ for all $w \in \Omega$. Put $G=1 / F$. Then clearly $G \in L^{\infty}(\Omega)$. We must show that $G \in A Q(\Omega)$, which by Theorem 7 is equivalent to showing that

$$
\left\|G \circ \varphi_{\lambda}-P\left(G \circ \varphi_{\lambda}\right)\right\|_{2} \rightarrow 0
$$

as $\lambda \rightarrow \partial \Omega$. Let $\left\{\lambda_{m}\right\}$ be a sequence in $\Omega$ tending to $\partial \Omega$. By the proof of Proposition 12, $\left\|F \circ \varphi_{\lambda_{m}}-\widetilde{F} \circ \varphi_{\lambda_{m}}\right\|_{2} \rightarrow 0$, and thus-by going to a subsequence -we may assume that $F \circ \varphi_{\lambda_{m}}-\widetilde{F} \circ \varphi_{\lambda_{m}} \rightarrow 0$ almost everywhere on $\Omega$ as $m \rightarrow \infty$. Now let $\left\{\lambda_{\alpha}\right\}$ a subnet of $\left\{\lambda_{m}\right\}$ such that $\varphi_{\lambda_{\alpha}} \rightarrow \varphi$ in $(\beta \Omega)^{\Omega}$ for some $\varphi \in \Phi$. By Proposition 12 we have $\widetilde{F} \in A Q(\Omega)$, thus the function $h=\widetilde{F}^{\beta} \circ \varphi \in H^{\infty}(\Omega)$. Since $\left\|F \circ \varphi_{\lambda_{\alpha}}-\widetilde{F} \circ \varphi_{\lambda_{\alpha}}\right\|_{2} \rightarrow 0$ we have $F \circ \varphi_{\lambda_{\alpha}} \rightarrow h$ in $L^{2}(\Omega, d V)$. Also note that $|h(w)| \geq \delta$ for all $w \in \Omega$. It follows from $\left\|G \circ \varphi_{\lambda_{\alpha}}-1 / h\right\|_{2} \leq\left\|F \circ \varphi_{\lambda_{\alpha}}-h\right\|_{2} / \delta^{2}$ that $\left\|G \circ \varphi_{\lambda_{\alpha}}-1 / h\right\|_{2} \rightarrow 0$. Applying $P$ we also get $\left\|P\left(G \circ \varphi_{\lambda_{\alpha}}\right)-1 / h\right\|_{2} \rightarrow 0$, thus $\left\|G \circ \varphi_{\lambda_{\alpha}}-P\left(G \circ \varphi_{\lambda_{\alpha}}\right)\right\|_{2} \rightarrow 0$. We conclude that $\left\|G \circ \varphi_{\lambda}-P\left(G \circ \varphi_{\lambda}\right)\right\|_{2} \rightarrow 0$ as $\lambda \rightarrow \partial \Omega$, and by Theorem $7, H_{G}$ is compact, i.e., $G \in A Q(\Omega)$.

It will be convenient to make use of the following well-known identity which gives a simple relationship between Toeplitz and Hankel operators:

$$
T_{g f}-T_{g} T_{f}=H_{\bar{g}}^{*} H_{f},
$$

for $f, g \in L^{\infty}(\Omega)$.

The following theorem describes the essential spectrum of the Toeplitz operators for which the corresponding Hankel operator is compact. 
Theorem 19. Let $f \in A Q(\Omega)$. Then

$$
\sigma_{e}\left(T_{f}\right)=\bigcap_{0<r<1} \operatorname{cl} \tilde{f}(\Omega \backslash r \Omega) .
$$

Proof. Let $f \in A Q(\Omega)$. We will divide the proof into several steps.

Step 1. Suppose that $\zeta \notin \operatorname{cl} f(\Omega \backslash r \Omega)$ for some $r \in(0,1)$. Define the function $g: \Omega \rightarrow \mathbb{C}$ by $g(z)=(f(z)-\zeta)^{-1}$ if $z \in \Omega \backslash r \Omega ; g(z)=1$ if $z \in r \Omega$. Then $g \in L^{\infty}(\Omega)$ and $g(f-\zeta)=\chi_{\Omega \backslash r \Omega}+(f-\zeta) \chi_{r \Omega}$, so that by (17) we have

$$
T_{g} T_{f-\zeta}=I+T_{(f-\zeta-1) \chi_{r \Omega}}-H_{\bar{g}}^{*} H_{f}
$$

Since both the operators $H_{f}$ and $T_{(f-\zeta-1) \chi_{r \Omega}}$ at the right-hand side of (18) are compact it follows that $T_{f-\zeta}+\mathscr{K}$ is left-invertible in the Calkin algebra $\mathscr{L}\left(L_{a}^{2}(\Omega)\right) / \mathscr{K}$. Reversing the roles of $f-\zeta$ and $g$ we also have

$$
T_{f-\zeta} T_{g}=I+T_{(f-\zeta-1) \chi_{r \Omega}}-H_{f}^{*} H_{g} .
$$

Put $F=(f-\zeta) \chi_{\Omega \backslash r \Omega}+\chi_{r \Omega}$. Then clearly $H_{F}$ is compact, i.e., $F \in A Q(\Omega)$. There is a $\delta>0$ such that $|F(w)| \geq \delta$ for all $w \in \Omega$. So by Lemma 18 , $1 / F \in A Q(\Omega)$. But $1 / F=g$, so $H_{g}$ is compact. It follows from (19) that $T_{f-\zeta}+\mathscr{K}$ is also right-invertible in $\mathscr{L}\left(L_{a}^{2}(\Omega)\right) / \mathscr{K}$. Thus $T_{f}-\zeta+\mathscr{K}=T_{f-\zeta}+\mathscr{K}$ is invertible in $\mathscr{L}\left(L_{a}^{2}(\Omega)\right) / \mathscr{K}$, so that $\zeta \notin \sigma_{e}\left(T_{f}\right)$. We conclude that $\sigma_{e}\left(T_{f}\right) \subset$ cl $f(\Omega \backslash r \Omega)$ for all $r \in(0,1)$.

Step 2. By Proposition 12, $T_{f-\widetilde{f}}$ is compact, thus $\sigma_{e}\left(T_{f}\right)=\sigma_{e}\left(T_{\widetilde{f}}\right)$, so that by Step $1, \sigma_{e}\left(T_{f}\right) \subset \operatorname{cl} \tilde{f}(\Omega \backslash r \Omega)$ for all $r \in(0,1)$.

Step 3. Now suppose that $\zeta \in \mathrm{cl} \tilde{f}(\Omega \backslash r \Omega)$ for all $r \in(0,1)$. Pick a net $\left\{\lambda_{\alpha}\right\}$ in $\Omega$ such that $\tilde{f}\left(\lambda_{\alpha}\right) \rightarrow \zeta, \lambda_{\alpha} \rightarrow \partial \Omega$ and $\varphi_{\lambda_{\alpha}} \rightarrow \varphi$ in $(\beta \Omega)^{\Omega}$ for some $\varphi \in \Phi$. Note that $\widetilde{f}^{\beta} \circ \varphi(0)=\zeta$. Since $\tilde{f}^{\beta} \circ \varphi$ is analytic on $\Omega$, we have $P\left(\overline{\tilde{f}}^{\beta} \circ \varphi\right)=P\left(\overline{\tilde{f}^{\beta} \circ \varphi}\right)=\overline{\tilde{f}^{\beta} \circ \varphi(0)}=\bar{\zeta}$, so that $P\left(\tilde{\tilde{f}}^{\beta} \circ \varphi-\bar{\zeta}\right) \equiv 0$. Hence $\left\|T_{\tilde{f}-\bar{\zeta}}\left(k_{\lambda_{\alpha}} /\left\|k_{\lambda_{\alpha}}\right\|_{2}\right)\right\|_{2}=\left\|P\left(\tilde{\tilde{f}}^{\beta} \circ \varphi_{\lambda_{\alpha}}-\bar{\zeta}\right)\right\|_{2} \rightarrow\left\|P\left(\tilde{\tilde{f}}^{\beta} \circ \varphi-\bar{\zeta}\right)\right\|_{2}=0$, and $T_{\bar{f}-\bar{\zeta}}+\mathscr{K}$ cannot be left-invertible in the Calkin algebra $\mathscr{L}\left(L_{a}^{2}(\Omega)\right) / \mathscr{K}$. Therefore $T_{\tilde{f}-\zeta}+\mathscr{K}$ is not right-invertible in the Calkin algebra, and thus $\zeta \in \sigma_{e}\left(T_{\tilde{f}}\right)=\sigma_{e}\left(T_{f}\right)$.

Remark. The above proof shows that for $f$ in $A Q(\Omega)$ the left-essential and the right-essential spectra coincide with the essential spectrum.

Corollary 20. Let $f \in A Q(\Omega)$. Then the essential spectrum $\sigma_{e}\left(T_{f}\right)$ is connected. Proof. Since the function $\tilde{f}$ is a bounded continuous function on $\Omega$, the sets cl $\tilde{f}(\Omega \backslash r \Omega)$ are compact and connected. By Theorem 19, $\sigma_{e}\left(T_{f}\right)$ is the intersection of a nested family of compact connected sets.

The essential norm of an operator is its distance to the compact operators, i.e., if $T$ is an operator in $\mathscr{L}\left(L_{a}^{2}(\Omega)\right)$, then its essential norm, denoted by $\|T\|_{e}$, is by definition the norm of the operator $T+\mathscr{K}$ in the Calkin algebra $\mathscr{L}\left(L_{a}^{2}(\Omega)\right) / \mathscr{K}$. The following corollary gives the essential norm for the Toeplitz operators on the Bergman space $L_{a}^{2}(\Omega)$ for which the corresponding Hankel operator is compact. 
Corollary 21. Let $f \in A Q(\Omega)$. Then

$$
\left\|T_{f}\right\|_{e}=\limsup _{w \rightarrow \partial \Omega}|\tilde{f}(w)| \text {. }
$$

Proof. Let $f \in A Q(\Omega)$. It is an easy consequence of identity (17) that $T_{f}^{*} T_{f}$ $T_{f} T_{f}^{*}=H_{\bar{f}}^{*} H_{\bar{f}}-H_{f}^{*} H_{f}$, which together with the fact that $H_{f}$ is compact implies that $T_{f}+\mathscr{K}$ is a hyponormal element of the Calkin algebra $\mathscr{L}\left(L_{a}^{2}(\Omega)\right) / \mathscr{K}$. By Stampfli's Theorem [9, III. Proposition 4.7], the norm of $T_{f}+\mathscr{K}$ is equal to its spectral radius $\max \left\{|\zeta|: \zeta \in \sigma_{e}\left(T_{f}\right)\right\}$. By Theorem 19 this quantity is equal to $\limsup _{w \rightarrow \partial \Omega}|\tilde{f}(w)|$.

To state yet another corollary of Theorem 19 we recall that an operator in $\mathscr{L}\left(L_{a}^{2}(\Omega)\right)$ is called Fredholm if its kernel has finite dimension and its range has finite codimension. It is a standard fact that $T$ in $\mathscr{L}\left(L_{a}^{2}(\Omega)\right)$ is Fredholm if and only if the operator $T+\mathscr{K}$ is invertible in the Calkin algebra $\mathscr{L}\left(L_{a}^{2}(\Omega)\right) / \mathscr{K}$.

Corollary 22. Let $f \in A Q(\Omega)$. Then $T_{f}$ is a Fredholm operator if and only if there exist $r \in(0,1)$ and $\delta>0$ such that $|\tilde{f}(w)|>\delta$ for every $w \in \Omega \backslash r \Omega$.

Proof. Observing that $T_{f}$ is Fredholm if and only if $0 \notin \sigma_{e}\left(T_{f}\right)$, the statement follows immediately from Theorem 19.

Recall that for a Fredholm operator $T$ in $\mathscr{L}\left(L_{a}^{2}(\Omega)\right)$ its index, denoted by ind $(T)$, is defined to be the difference between the dimension of the kernel of $T$ and the codimension of the range of $T$. In addition to the above characterization of the Toeplitz operators that are Fredholm, we have a formula for the index of such Toeplitz operators. Before stating and proving this index theorem, we consider a special case in the following lemma.

Lemma 23. Let $F \in A Q(\Omega) \cap B C(\Omega)$ be bounded away from 0 on $\Omega, 0<r<1$, and suppose that $F$ agrees with $\tilde{f} h$ on $\Omega \backslash r \Omega$ where $f \in A Q(\Omega)$ and $h \mid \Omega \backslash r \Omega$ extends continuously to $\operatorname{cl}(\Omega \backslash r \Omega)$. Then $T_{F}$ is Fredholm, and $\operatorname{ind}\left(T_{F}\right)=0$.

Proof. Suppose $F \in A Q(\Omega) \cap B C(\Omega)$ is bounded away from 0 on $\Omega$ and $F$ agrees with $\tilde{f} h$ on $\Omega \backslash r \Omega$ where $f \in A Q(\Omega)$ and $h \mid \Omega \backslash r \Omega$ extends continuously to $\operatorname{cl}(\Omega \backslash r \Omega)$. Let $\delta>0$ be such that $|F(w)| \geq \delta$ for all $w \in \Omega$. By Step 1 of

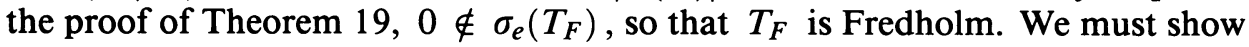
that $\operatorname{ind}\left(T_{F}\right)=0$. For $m \in \mathbb{N}$ let $G$ be a continuous function on $\Omega$ such that $G^{m}=F$. Since $F$ is bounded on $\Omega$, so is the function $G$. We assert that $G$ is in $A Q(\Omega)$. Assuming that $G \in A Q(\Omega)$, by (17) we have $T_{F}=\left(T_{G}\right)^{m}+K$, where $K$ is a compact operator. Since $|G(w)| \geq \delta^{1 / m}$ for all $w \in \Omega$, also $T_{G}$ is Fredholm, and it follows that $\operatorname{ind}\left(T_{F}\right)=m \operatorname{ind}\left(T_{G}\right)$. Thus $\operatorname{ind}\left(T_{F}\right)$ is divisible by any $m \in \mathbb{N}$, and we conclude that $\operatorname{ind}\left(T_{F}\right)=0$.

It remains to show that $G$ is in $A Q(\Omega)$. Let $\left\{\lambda_{\alpha}\right\}$ be a net in $\Omega$ such that $\lambda_{\alpha} \rightarrow \partial \Omega$ and $\varphi_{\lambda_{\alpha}} \rightarrow \varphi$ in $(\beta \Omega)^{\Omega}$ for some $\varphi \in \Phi$. Then $\tilde{f} \circ \varphi_{\lambda_{\alpha}} \rightarrow \tilde{f}^{\beta} \circ \varphi$ uniformly on compact subsets of $\Omega$. We will show that also $G \circ \varphi_{\lambda_{\alpha}} \rightarrow G^{\beta} \circ \varphi$ uniformly on compact subsets of $\Omega$. It will then follow that $F \circ \varphi_{\lambda_{\alpha}} \rightarrow F^{\beta} \circ \varphi$ uniformly on compact subsets of $\Omega$. As in the proof of Theorem 14 this implies that $F^{\beta} \circ \varphi$ is analytic on $\Omega$. Since $G^{\beta} \circ \varphi$ is continuous on $\Omega$, and $\left(G^{\beta} \circ \varphi\right)^{m}=F^{\beta} \circ \varphi$ is analytic on $\Omega$, we have that $G^{\beta} \circ \varphi$ is analytic on $\Omega$. 
Also $G \circ \varphi_{\lambda_{\alpha}} \rightarrow G^{\beta} \circ \varphi$ in $L^{2}(\Omega, d V)$, and thus $\left\|G \circ \varphi_{\lambda_{\alpha}}-P\left(G \circ \varphi_{\lambda_{\alpha}}\right)\right\|_{2} \rightarrow 0$, so that we can conclude that $H_{G}$ is compact.

To finish the proof we have to show that $G \circ \varphi_{\lambda_{\alpha}} \rightarrow G^{\beta} \circ \varphi$ uniformly on compact subsets of $\Omega$. We have pointwise convergence, so by a standard argument it suffices to show that for arbitrary $\varepsilon>0$ and $w \in \Omega$ there are $\rho>0$ and an index $\alpha_{0}$ such that for every $\alpha \geq \alpha_{0}$ we have: $\left|G \circ \varphi_{\lambda_{\alpha}}(z)-G \circ \varphi_{\lambda_{\alpha}}(w)\right|<\varepsilon$ whenever $b(z, w)<\rho$. Since $h$ is bounded away from 0 on $\operatorname{cl}(\Omega \backslash r \Omega)$ we can choose a continuous function $k$ on $\operatorname{cl}(\Omega \backslash r \Omega)$ so that $k^{m}=h$ on $\operatorname{cl}(\Omega \backslash r \Omega)$. Putting $g=G / k$ on $\Omega \backslash r \Omega$, we then also have $g^{m}=\tilde{f}$ on $\Omega \backslash r \Omega$. Now let $\varepsilon>0$ and $w \in \Omega$ be given. Note that $\eta=\inf \{|\tilde{f}(z)|: z \in \Omega \backslash r \Omega\}$ is positive; let $\rho=\frac{1}{2} m \varepsilon \eta^{1-1 / m} /\left(C_{f}\|k\|_{\infty}\right)$, where $C_{f}$ is the constant for which inequality (15) holds. Let $E(w, \rho)=\{z \in \Omega: b(z, w)<\rho\}$. Since $\varphi_{\lambda_{\alpha}}(w) \rightarrow \partial \Omega$ we can choose an index $\alpha_{0}$ (depending on $w$ ) such that for $\alpha \geq \alpha_{0}: \varphi_{\lambda_{\alpha}}(E(w, \rho)) \subset \Omega \backslash r \Omega$. Now let $z \in E(w, \rho)$. Then taking for $\sigma:[0,1] \rightarrow \Omega$ a geodesic from $w$ to $z$ in the Bergman metric $b$ it follows from (15) that

$$
\begin{aligned}
\left|g\left(\varphi_{\lambda_{\alpha}}(z)\right)-g\left(\varphi_{\lambda_{\alpha}}(w)\right)\right| & \leq \int_{0}^{1}\left|\frac{d}{d t} g\left(\varphi_{\lambda_{\alpha}}(\sigma(t))\right)\right| d t \\
& =\int_{0}^{1} \frac{1}{m}\left|\widetilde{f}\left(\varphi_{\lambda_{\alpha}}(\sigma(t))\right)^{(1-m) / m} \frac{d}{d t} \widetilde{f}\left(\varphi_{\lambda_{\alpha}}(\sigma(t))\right)\right| d t \\
& \leq \frac{1}{m} \eta^{(1-m) / m} C_{f} b\left(\varphi_{\lambda_{\alpha}}(z), \varphi_{\lambda_{\alpha}}(w)\right) \\
& =\frac{1}{m} \eta^{(1-m) / m} C_{f} b(z, w)<\varepsilon /\left(2\|k\|_{\infty}\right)
\end{aligned}
$$

whenever $z \in E(w, \rho)$ and $\alpha \geq \alpha_{0}$. Since $k$ is uniformly continuous on $\Omega \backslash r \Omega$ we may assume that furthermore $\left|k\left(\varphi_{\lambda_{\alpha}}(z)\right)-k\left(\varphi_{\lambda_{\alpha}}(w)\right)\right|<\varepsilon /\left(2\|g\|_{\infty}\right)$ whenever $z \in E(w, \rho)$ and $\alpha \geq \alpha_{0}$. It follows immediately that $\mid G\left(\varphi_{\lambda_{\alpha}}(z)\right)-$ $G\left(\varphi_{\lambda_{\alpha}}(w)\right) \mid<\varepsilon$ whenever $z \in E(w, \rho)$ and $\alpha \geq \alpha_{0}$, and our claim is proved.

Now we are in a position to prove the following index theorem.

Theorem 24. Let $f \in A Q(\Omega)$ and suppose that $T_{f}$ is a Fredholm operator. Then

$$
\operatorname{ind}\left(T_{f}\right)= \begin{cases}- \text { winding number of }\left.\widetilde{f}\right|_{\{|z|=r\}} & \\ \text { for some } r<1 \text { close to } 1 & \text { if } n=1 ; \\ 0 & \text { if } n \geq 2 .\end{cases}
$$

Proof. Let $f \in A Q(\Omega)$ be such that $T_{f}$ is Fredholm. By Corollary 17, there is an $r \in(0,1)$ and $\delta>0$ such that $|\widetilde{f}(w)| \geq \delta$ for all $w \in \Omega \backslash r \Omega$. Also, we have proved that $T_{f}=T_{\widetilde{f}}+K$, for some compact operator $K$ (Proposition 12). Thus $T_{\widetilde{f}}$ is Fredholm and $\operatorname{ind}\left(T_{f}\right)=\operatorname{ind}\left(T_{\widetilde{f}}\right)$. Note that $\tilde{f} \in A Q(\Omega) \cap B C(\Omega)$. Now consider the following two cases.

Case 1: $n=1$. Suppose that $\widetilde{f}$ has winding number $m$ on $|z|=r$. Then the function $\tilde{f}(z)(|z| / z)^{m}$ on $\mathbb{D} \backslash r \mathbb{D}$ extends to a function $F \in B C(\mathbb{D})$ bounded away from 0 on $\mathbb{D}$. Let $g$ be the function defined by

$$
g(z)= \begin{cases}|z| / z & \text { if } z \in \mathbb{D} \backslash r \mathbb{D} ; \\ 1 & \text { if } z \in r \mathbb{D} .\end{cases}
$$


One easily verifies that $H_{|z|}$ is compact, and using this it follows that $H_{g}$ is compact, i.e., $g \in A Q(\mathbb{D})$. Using that $A Q(\mathbb{D})$ is an algebra (and if necessary Lemma 18), we see that $g^{m} \in A Q(\mathbb{D})$, hence $\tilde{f} g^{m} \in A Q(\mathbb{D})$. Since $F$ agrees with $\tilde{f} g^{m}$ on $\mathbb{D} \backslash r \mathbb{D}$, also $F \in A Q(\mathbb{D})$. By Lemma 23 , ind $\left(T_{F}\right)=0$. Using (17), $T_{F}=T_{\widetilde{f}}\left(T_{g}\right)^{m}+K$, for some compact operator $K$, and thus $\operatorname{ind}\left(T_{F}\right)=$ $\operatorname{ind}\left(T_{\widetilde{f}}\right)+m \operatorname{ind}\left(T_{g}\right)$. We get $\operatorname{ind}\left(T_{\widetilde{f}}\right)=-m \operatorname{ind}\left(T_{g}\right)$, and it remains to show that $\operatorname{ind}\left(T_{g}\right)=1$. Let the function $h$ be defined by

$$
h(z)= \begin{cases}|z| & \text { if } z \in \mathbb{D} \backslash r \mathbb{D} \\ r & \text { if } z \in r \mathbb{D} .\end{cases}
$$

Then $z g$ and $h$ differ only on $r \mathbb{D}$, so that $\operatorname{ind}\left(T_{z g}\right)=\operatorname{ind}\left(T_{h}\right)$. But by Lemma 23 , $\operatorname{ind}\left(T_{h}\right)=0$. Also, ind $\left(T_{z g}\right)=\operatorname{ind}\left(T_{z}\right)+\operatorname{ind}\left(T_{g}\right)$. Thus $\operatorname{ind}\left(T_{g}\right)=$ $-\operatorname{ind}\left(T_{z}\right)$. It is easy to see that $\operatorname{ind}\left(T_{z}\right)=-1$, so that $\operatorname{ind}\left(T_{g}\right)=1$, and we are done with this case.

Case 2: $n \geq 2$. In this case there is a bounded continuous function $g$ on $\Omega$ such that $g(w)=\tilde{f}(w)$ for $w \in \Omega \backslash r \Omega$, and $g$ is bounded away from 0 on all of $\Omega$. Since $g-\widetilde{f}$ is supported on $r \Omega, T_{g}-T_{\widetilde{f}}$ is compact (by Corollary 9). Thus $T_{g}$ is also Fredholm, and $\operatorname{ind}\left(T_{\widetilde{f}}\right)=\operatorname{ind}\left(T_{g}\right)$. By Lemma $23, \operatorname{ind}\left(T_{g}\right)=0$, hence $\operatorname{ind}\left(T_{f}\right)=0$.

\section{TOEPLitZ AND HANKel OPERATORS WITH SPECIAL SYMBols}

Let $\mathscr{U}(\Omega)$ be the norm-closed subalgebra of $L^{\infty}(\Omega)$ generated by the bounded analytic functions on $\Omega$ and their complex conjugates. In this section we will characterize more precisely the functions $f$ in $\mathscr{U}(\Omega)$ such that $T_{f}$ or $H_{f}$ is compact. First we describe an alternative way of looking at the algebra $\mathscr{U}(\Omega)$. Let $\mathscr{M}$ denote the maximal ideal space of $H^{\infty}(\Omega)$, which is defined to be the set of all multiplicative linear functions from $H^{\infty}(\Omega)$ onto the complex plane. The space $\mathscr{M}$ endowed with the weak-star topology inherited from the dual of $H^{\infty}(\Omega)$ is a compact Hausdorff space. Using the Gelfand transform we can think of $H^{\infty}(\Omega)$ as a subset of $C(\mathscr{M})$, the algebra of continuous complex-valued functions on $\mathscr{M}$. For $\lambda$ in $\Omega$ the evaluation $f \mapsto f(\lambda)$ is a multiplicative linear functional on $H^{\infty}(\Omega)$, thus we can think of $\Omega$ as a subset of $\mathscr{M}$. We note that Lemma 4.4 in [12] can be extended to show that the algebra $\mathscr{U}(\Omega)$ is precisely the set of functions on $\Omega$ that extend continuously to the maximal ideal space of $H^{\infty}(\Omega)$.

For the unit ball $\mathbb{B}_{n}$, the algebra $\mathscr{U}\left(\mathbb{B}_{n}\right)$ is furthermore equal to the normclosed subalgebra of $L^{\infty}(\Omega)$ generated by the bounded pluriharmonic functions on $\mathbb{B}_{n}$ (see [22]).

Clearly $\mathscr{U}(\Omega)$ is contained in $B C(\Omega)$. The following lemma should be compared with Lemma 13.

Lemma 25. Let $f \in \mathscr{U}(\Omega)$, and let $\left\{w_{\alpha}\right\}$ be a net in $\Omega$ tending to $\partial \Omega$ such that $\varphi_{w_{\alpha}} \rightarrow \varphi$ in $(\beta \Omega)^{\Omega}$. Then $f \circ \varphi_{w_{\alpha}}$ converges to $f^{\beta} \circ \varphi$ uniformly on each compact subset of $\Omega$.

Proof. For $g_{1}, \ldots, g_{N}, h_{1}, \ldots, h_{N} \in H^{\infty}(\Omega)$, we have $\widetilde{h}_{j}=h_{j}$ and $\tilde{g}_{j}=$ $\bar{g}_{j}(j=1, \ldots, n)$ so that by Lemma 13 the statement is true for each of the 
functions $\bar{g}_{j}$ and $h_{j}$, and thus for $u=\bar{g}_{1} h_{1}+\cdots+\bar{g}_{N} h_{N}$. Since these functions $u$ are dense in $\mathscr{U}(\Omega)$ the statement holds for every $f \in \mathscr{U}(\Omega)$.

As a consequence of the above lemma we see that for $f \in \mathscr{U}(\Omega)$, and $\left\{w_{\alpha}\right\}$ a net in $\Omega$ tending to $\partial \Omega$ such that $\varphi_{w_{\alpha}} \rightarrow \varphi$ in $(\beta \Omega)^{\Omega}$, we have $f \circ \varphi_{w_{\alpha}} \rightarrow f^{\beta} \circ \varphi$ in $L^{2}(\Omega, d V)$; from this we obtain the following version of Theorem 7 .

Theorem 26. For $f \in \mathscr{U}(\Omega)$ we have:

(1) $H_{f}$ is compact $\Leftrightarrow f \in A O \Phi$;

(2) both $H_{f}$ and $H_{\bar{f}}$ are compact $\Leftrightarrow f \in C O \Phi$.

We will use the above theorem to characterize the symbols in $\mathscr{U}(\Omega)$ for which the Toeplitz operator is compact. We have the following generalization of Proposition 5 in [15].

Theorem 27. Let $f \in \mathscr{U}(\Omega)$. Then $T_{f}$ is compact if and only if $f$ is continuously extendable to $\mathrm{cl} \Omega$ with $f \mid \partial \Omega=0$.

Before we prove this theorem we recall the following algebraic properties of Toeplitz operators:

$$
T_{f h}=T_{f} T_{h} \text { and } T_{\bar{g} f}=T_{g}^{*} T_{f} \text {, for all } f \in L^{\infty}(\Omega) \text { and } g, h \in H^{\infty}(\Omega) .
$$

The following lemma is a straightforward generalization of a lemma in [15]. For completeness we will give a proof.

Lemma 28. The set $\left\{f \in \mathscr{U}(\Omega): T_{f}\right.$ is compact $\}$ is a closed ideal in $\mathscr{U}(\Omega)$. Proof. Put $\mathscr{I}=\left\{f \in \mathscr{U}(\Omega): T_{f}\right.$ is compact $\}$. It is clear that $\mathscr{I}$ is a closed linear subspace of $\mathscr{U}(\Omega)$. To show that it is an ideal, suppose that $f \in \mathscr{I}$. Let $g_{1}, \ldots, g_{N}$ and $h_{1}, \ldots, h_{N}$ be in $H^{\infty}(\Omega)$, and set $u=\bar{g}_{1} h_{1}+\cdots+\bar{g}_{N} h_{N}$. Then, using (20) we have $T_{f u}=T_{g_{1}}^{*} T_{f} T_{h_{1}}+\cdots+T_{g_{N}}^{*} T_{f} T_{h_{N}}$, and thus $T_{f u}$ is compact, i.e., $f u \in \mathscr{I}$. Functions of the above form are dense in $\mathscr{U}(\Omega)$, so $\mathscr{I}$ is an ideal in $\mathscr{U}(\Omega)$.

Proof of Theorem 27. If $f$ is continuous on $\operatorname{cl} \Omega$ and $f \mid \partial \Omega \equiv 0$, then $\left\|f \circ \varphi_{\lambda}\right\|_{2} \rightarrow 0$ as $\lambda \rightarrow \partial \Omega$ (by the Lebesgue Dominated Convergence Theorem), so that certainly $\left\|P\left(f \circ \varphi_{\lambda}\right)\right\|_{2} \rightarrow 0$ as $\lambda \rightarrow \partial \Omega$, and thus $T_{f}$ is compact.

Conversely, suppose that $f \in \mathscr{U}(\Omega)$ and $T_{f}$ is compact. Since $\bar{f} \in \mathscr{U}(\Omega)$, by Lemma 28 also $T_{|f|^{2}}$ is compact, thus $|\widetilde{f}|^{2}(\lambda) \rightarrow 0$ as $\lambda \rightarrow \partial \Omega$, and by Corollary 8 the operator $M_{f}$ is compact. It follows that both $H_{f}$ and $H_{\bar{f}}$ are compact. By Theorem 26, $f \in C O \Phi$, i.e., $f^{\beta} \circ \varphi$ is constant for every $\varphi \in \Phi$. The compactness of $T_{f}$ also implies that $P\left(f \circ \varphi_{\lambda}\right)(0)=\tilde{f}(\lambda) \rightarrow 0$ as $\lambda \rightarrow \partial \Omega$. This and the definition of $\Phi$ show that for a $\varphi \in \Phi, P\left(f^{\beta} \circ \varphi\right)(0)=0$, which combined with the fact that $f^{\beta} \circ \varphi$ is constant gives us that $f^{\beta} \circ \varphi \equiv 0$. It is now easily seen that necessarily $f(\lambda) \rightarrow 0$ as $\lambda \rightarrow \partial \Omega$.

Using Theorem 6 we can also give another proof of Theorem 27.

Second Proof of Theorem 27. Suppose that $T_{f}$ is compact for $f \in \mathscr{U}(\Omega)$. Take $g, h \in H^{\infty}(\Omega)$. Since $T_{f g}=T_{f} T_{g}$ is compact, using Lemma 25 and Theorem 6 , we conclude that $P\left(\left(f^{\beta} \circ \varphi\right)\left(g^{\beta} \circ \varphi\right)\right) \equiv 0$ for every $\varphi \in \Phi$. Thus we have $\left\langle\left(h^{\beta} \circ \varphi\right)\left(\bar{g}^{\beta} \circ \varphi\right), f^{\beta} \circ \varphi\right\rangle=\left\langle h^{\beta} \circ \varphi, P\left(\left(f^{\beta} \circ \varphi\right)\left(g^{\beta} \circ \varphi\right)\right)\right\rangle=0$. It follows that $\left\langle u^{\beta} \circ \varphi, f^{\beta} \circ \varphi\right\rangle=0$ for every function $u=\bar{g}_{1} h_{1}+\cdots+\bar{g}_{N} h_{N}$, where $g_{1}, \ldots, g_{N}$ 
and $h_{1}, \ldots, h_{N}$ are in $H^{\infty}(\Omega)$. Using that the functions $u$ of the above form are dense in $\mathscr{U}(\Omega)$, we get $\left\langle f^{\beta} \circ \varphi, f^{\beta} \circ \varphi\right\rangle=0$, so that $f^{\beta} \circ \varphi \equiv 0$.

More precise descriptions for compactness of Hankel operators with symbols in $\mathscr{U}(\Omega)$ are in the following theorems. In these theorems we use the notation $|E|$ to denote the normalized Lebesgue volume measure of a Lebesgue measurable set $E$ contained in $\Omega$.

Theorem 29. Let $f \in \mathscr{U}(\Omega), 0<r<1$, and $1<p<\infty$. Then the following statements are equivalent:

(a) $H_{f}$ is compact;

(b) $f \in A O \Phi$;

(c) distance $\left(f \mid \Omega(w, r), H^{\infty}(\Omega(w, r))\right) \rightarrow 0$ as $w \rightarrow \partial \Omega$;

(d) $\left\|f \circ \varphi_{w}-P\left(f \circ \varphi_{w}\right)\right\|_{p} \rightarrow 0$ as $w \rightarrow \partial \Omega$;

(e) $(1 /|\Omega(w, r)|) \int_{\Omega(w, r)}\left|f-P\left(f \circ \varphi_{w}\right) \circ \varphi_{w}\right|^{p} d V \rightarrow 0$ as $w \rightarrow \partial \Omega$.

Proof. We have already shown the equivalence of statements (a), (b), and (d).

Proof that $(\mathrm{a}) \Rightarrow(\mathrm{c})$. Suppose $H_{f}$ is compact. By Proposition 12 also $H_{\tilde{f}}$ is compact, and according to Proposition 16 we have

$$
\operatorname{distance}\left(\tilde{f} \mid \Omega(w, r), H^{\infty}(\Omega(w, r))\right) \rightarrow 0 \quad \text { as } w \rightarrow \partial \Omega .
$$

It follows from the analyticity of $f \circ \varphi$ that $\tilde{f} \circ \varphi=f \circ \varphi$ for every $\varphi \in \Phi$. Using Lemmas 13 and 25 this implies that

$$
\begin{aligned}
\sup \{|f(z)-\tilde{f}(z)|: & z \in \Omega(w, r)\} \\
& =\sup \left\{\left|\left(f \circ \varphi_{w}\right)(u)-\left(\tilde{f} \circ \varphi_{w}\right)(u)\right|: u \in r \Omega\right\} \rightarrow 0 .
\end{aligned}
$$

Hence distance $\left(f \mid \Omega(w, r), H^{\infty}(\Omega(w, r))\right) \rightarrow 0$ as $w \rightarrow \partial \Omega$.

The proof that (c) $\Rightarrow$ (b) is the same as the implication (iii) $\Rightarrow$ (i) in Proposition 16.

Proof that $(d) \Rightarrow(e)$. Using the same arguments as in $\S 2$ of [8] it is easy to show that there is a positive constant $C_{r}$ such that for all $w \in \Omega$ and every $z \in \Omega(w, r)$ :

$$
\begin{gathered}
C_{r}^{-1} k_{w}(w) \leq\left|k_{w}(z)\right| \leq C_{r} k_{w}(w), \quad \text { and } \\
C_{r}^{-1} k_{w}(w) \leq|\Omega(w, r)|^{-1} \leq C_{r} k_{w}(w) .
\end{gathered}
$$

Using these inequalities and change-of-variable formula (3) we have

$$
\begin{gathered}
\frac{1}{|\Omega(w, r)|} \int_{\Omega(w, r)}\left|f-P\left(f \circ \varphi_{w}\right) \circ \varphi_{w}\right|^{p} d V \\
\quad \leq C \int_{r \Omega}\left|f \circ \varphi_{w}-P\left(f \circ \varphi_{w}\right)\right|^{p} d V \\
\leq C\left\|f \circ \varphi_{w}-P\left(f \circ \varphi_{w}\right)\right\|_{p}^{p}
\end{gathered}
$$

and the implication follows.

Proof that $(\mathrm{e}) \Rightarrow(\mathrm{b})$. Let $\varphi \in \Phi$. Choose a net $\left\{w_{\alpha}\right\}$ in $\Omega$ tending to $\partial \Omega$ such that $\varphi_{w_{\alpha}} \rightarrow \varphi$ in $(\beta \Omega)^{\Omega}$. It

$$
\begin{aligned}
\left|P\left(f \circ \varphi_{w_{\alpha}}\right)(z)-P\left(f^{\beta} \circ \varphi\right)(z)\right| & =\left|\left\langle f \circ \varphi_{w_{\alpha}}-f^{\beta} \circ \varphi, k_{z}\right\rangle\right| \\
& \leq\left\|f \circ \varphi_{w_{\alpha}}-f^{\beta} \circ \varphi\right\|_{2} k_{z}(z)^{1 / 2}
\end{aligned}
$$


that $P\left(f \circ \varphi_{w_{\alpha}}\right) \rightarrow P\left(f^{\beta} \circ \varphi\right)$ uniformly on $r \Omega$, thus

$$
\int_{r \Omega}\left|f \circ \varphi_{w_{\alpha}}-P\left(f \circ \varphi_{w_{\alpha}}\right)\right|^{p} d V \rightarrow \int_{r \Omega}\left|f^{\beta} \circ \varphi-P\left(f^{\beta} \circ \varphi\right)\right|^{p} d V .
$$

On the other hand, using the above inequalities as well as change-of-variable formula (3) we have

$$
\begin{aligned}
& \int_{r \Omega}\left|f \circ \varphi_{w}-P\left(f \circ \varphi_{w}\right)\right|^{p} d V \\
& \quad \leq C \frac{1}{|\Omega(w, r)|} \int_{\Omega(w, r)}\left|f-P\left(f \circ \varphi_{w}\right) \circ \varphi_{w}\right|^{p} d V,
\end{aligned}
$$

thus $\int_{r \Omega}\left|f^{\beta} \circ \varphi-P\left(f^{\beta} \circ \varphi\right)\right|^{p} d V=0$, and we conclude that $f^{\beta} \circ \varphi=P\left(f^{\beta} \circ \varphi\right)$ on $r \Omega$, thus $f^{\beta} \circ \varphi$ is analytic on $r \Omega$. As before we deduce that $f^{\beta} \circ \varphi$ is analytic on all of $\Omega$. This for every $\varphi \in \Phi$, and it follows that $f \in A O \Phi$.

Theorem 30. Let $f \in \mathscr{U}(\Omega)$. Then the following statements are equivalent:

(a) $H_{f}$ and $H_{\bar{f}}$ are compact;

(b) $f \in C O \Phi$;

(c) $\sup \{|f(z)-f(w)|: z \in \Omega(w, r)\} \rightarrow 0$ as $w \rightarrow \partial \Omega$;

(d) $\left\|f \circ \varphi_{w}-\tilde{f}(w)\right\|_{p} \rightarrow 0$ as $w \rightarrow \partial \Omega$;

(e) $1 /(|\Omega(w, r)|) \int_{\Omega(w, r)}|f-\tilde{f}(w)|^{p} d V \rightarrow 0$ as $w \rightarrow \partial \Omega$.

Proof. We have already shown the equivalence of statements (a), (b), and (d).

Proof that $(\mathrm{a}) \Rightarrow(\mathrm{c})$. Suppose $H_{f}$ and $H_{\bar{f}}$ are compact. By Proposition 12 also $H_{\widetilde{f}}$ and $H_{\widetilde{\tilde{f}}}$ are compact, and according to Proposition 17 we have $\sup \{|\tilde{f}(z)-\tilde{f}(w)|: \quad z \in \Omega(w, r)\} \rightarrow 0$ as $w \rightarrow \partial \Omega$. As in the above proof, we also have $\sup \{|f(z)-\tilde{f}(z)|: \quad z \in \Omega(w, r)\} \rightarrow 0$ as $w \rightarrow \partial \Omega$, hence $\sup \{|f(z)-f(w)|: \quad z \in \Omega(w, r)\} \rightarrow 0$ as $w \rightarrow \partial \Omega$.

The proof that (c) $\Rightarrow$ (b) is the same as the implication (iii) $\Rightarrow$ (i) in Proposition 17.

Proof that $(\mathrm{d}) \Rightarrow(\mathrm{e})$. As in the above proof, there is a constant $C$ such that for every $w \in \Omega$ :

$$
\frac{1}{|\Omega(w, r)|} \int_{\Omega(w, r)}|f-\widetilde{f}(w)|^{p} d V \leq C \| f \circ \varphi_{w}-\left.\tilde{f}(w)\right|_{p} ^{p},
$$

and the statement follows.

Proof that $(\mathrm{e}) \Rightarrow(\mathrm{b})$. Let $\varphi \in \Phi$. Choose a net $\left\{w_{\alpha}\right\}$ in $\Omega$ tending to $\partial \Omega$ such that $\varphi_{w_{\alpha}} \rightarrow \varphi$ in $(\beta \Omega)^{\Omega}$. Using Lemma 25 we have

$$
\int_{r \Omega}\left|f \circ \varphi_{w_{\alpha}}-\tilde{f}\left(w_{\alpha}\right)\right|^{p} d V \rightarrow \int_{r \Omega}\left|f^{\beta} \circ \varphi-\left(f^{\beta} \circ \varphi\right)(0)\right|^{p} d V .
$$

On the other hand, as in the above proof, there is a constant $C$ such that for every $w \in \Omega$ :

$$
\int_{r \Omega}\left|f \circ \varphi_{w}-\tilde{f}(w)\right|^{p} d V \leq C \frac{1}{|\Omega(w, r)|} \int_{\Omega(w, r)}|f-\tilde{f}(w)|^{p} d V,
$$

thus $\int_{r \Omega}\left|f^{\beta} \circ \varphi-\left(f^{\beta} \circ \varphi\right)(0)\right|^{p} d V=0$, and we conclude that $f^{\beta} \circ \varphi=\left(f^{\beta} \circ \varphi\right)(0)$ on $r \Omega$, thus $f^{\beta} \circ \varphi$ is constant on $r \Omega$. As before we deduce that $f^{\beta} \circ \varphi$ is constant on all of $\Omega$. This for every $\varphi \in \Phi$, and it follows that $f \in C O \Phi$. 


\section{REMARKS AND OPEN QUESTIONS}

In this section we will make some remarks and formulate some open questions.

1. The results in this paper can be extended somewhat. If $\Omega$ is a bounded symmetric domain in $\mathbb{C}^{n}$ and $0 \in \Omega$, then for every $\lambda \in \Omega$ there is an automorphism $\varphi_{\lambda}$ on $\Omega$ such that $\varphi_{\lambda}(\lambda)=0$ and $\varphi_{\lambda} \circ \varphi_{\lambda}=\mathrm{id}_{\Omega}$. It is then easy to see that Proposition 1 extends to arbitrary bounded symmetric domains $\Omega$. In trying to extend the results in $\S 3$ one would need to know when the quantities $M_{q, \varepsilon}(\Omega)$ are finite. In the proof of Lemma 2 we used the ForelliRudin estimates on the reproducing kernels for the unit ball. Recently these estimates have been extended somewhat in [10]. Unfortunately, the extended Forelli-Rudin estimates obtained in [10] are only valid for a certain range of parameters (depending on the domain), and do not give Lemma 2 for the domain. However, for the rank 2 domain $\Omega_{3}$ in $\mathbb{C}^{3}$ consisting of $2 \times 2$ contractive symmetric matrices it follows from the generalized Forelli-Rudin inequalities in [10] that $M_{q, \varepsilon}\left(\Omega_{3}\right)<\infty$ for arbitrary small $\varepsilon>0$ and $q>1$ sufficiently close to 1 ; in fact, there are sequences $\left(q_{N}\right)$ in $(1, \infty)$ and $\left(\varepsilon_{N}\right)$ in $(0, \infty)$ converging to 1 and 0 respectively such that for every positive integer $N$ we have $M_{q_{N}, \varepsilon_{N}}\left(\Omega_{3}\right)<\infty$. Using that the reproducing kernel of a product of domains is the product of the reproducing kernels, it follows that for $\Omega$ a finite product of unit balls and copies of $\Omega_{3}$, the quantity $M_{q, \varepsilon}(\Omega)$ is finite for arbitrary small $\varepsilon>0$ and $q>1$ sufficiently close to 1 . In the proof of Theorems 6 and 7 it was also needed that the Bergman projection is $L^{p}$-bounded for arbitrary $p>1$; we do not know whether this is true for arbitrary bounded symmetric domains, but in the case of domain $\Omega_{3}$ it follows easily from the generalized Forelli-Rudin inequalities in [10]. Thus Theorems 6 and 7 hold for finite products of unit balls and copies of $\Omega_{3}$.

Question 1. Do Theorems 6 and 7 hold for arbitrary bounded symmetric domains in $\mathbb{C}^{n}$ ?

Remark. The descriptions of the algebras $A O \Phi$ and $C O \Phi$ in $\S 6$-equivalences (b)-(f) in Theorems 29 and 30 respectively-do hold for arbitrary bounded symmetric domains in $\mathbb{C}^{n}$.

2. We note that in [19] the first author has used the method of $\S 3$ to prove that the analogous results hold for Hankel and Toeplitz operators on the Fock space (Segal-Bargmann space), the space of entire functions on $\mathbb{C}^{n}$ which are square-integrable with respect to Gaussian measure on $\mathbb{C}^{n}$, thus providing an alternate approach to the work of C. A. Berger and L. A. Coburn [7]. It would be interesting to find other settings for which the results hold.

3. In this paper we defined $A Q(\Omega)=\left\{f \in L^{\infty}(\Omega): H_{f}\right.$ is compact $\}$. Although Theorem 7 characterizes the algebra $A Q(\Omega)$ and Proposition 12 tells us that $A Q(\Omega)$ is decomposed as $A Q(\Omega)=\mathscr{I}(\Omega)+A Q(\Omega) \cap B C(\Omega)$ where $\mathscr{J}(\Omega)$ denotes the ideal $\left\{g \in L^{\infty}(\Omega):|\widetilde{g}|^{2}(\lambda) \rightarrow 0\right.$ as $\left.\lambda \rightarrow \partial \Omega\right\}$, it would be nice to have more precise descriptions of the algebra $A Q(\Omega)$. The $C^{*}$-algebra $Q(\Omega)=\left\{f \in L^{\infty}(\Omega): H_{f}\right.$ and $H_{\bar{f}}$ are compact $\}$ has been studied extensively in [6, 8, and 23]. It is obvious that $H^{\infty}(\Omega)+Q(\Omega) \subset A Q(\Omega)$.

Question 2. Is $A Q(\Omega)$ equal to $H^{\infty}(\Omega)+Q(\Omega)$ ? 
This question extends Sheldon Axler and Pamela Gorkin's question raised in [5] whether for the unit disk $\mathbb{D}$ one has $A O P=H^{\infty}(\mathbb{D})+C O P$ ?

\section{REFERENCES}

1. Sheldon Axler, Hankel operators on Bergman spaces, Linear and Complex Analysis Problem Book, edited by V. P. Havin, S. V. Hruščëv, and N. K. Nikol'skiī, Lecture Notes in Math., vol. 1043, Springer, Berlin, 1984, pp. 262-263.

2. _ The Bergman space, the Bloch space, and commutators of multiplication operators, Duke Math. J. 53 (1986), 315-332.

3. In Informal notes on $C O P$ and $A O P$, unpublished manuscript.

4. Sheldon Axler, John B. Conway, and Gerald McDonald, Toeplitz operators on Bergman spaces, Canad. J. Math. 34 (1982), 466-483.

5. Sheldon Axler and Pamela Gorkin, Algebras on the disk and doubly commuting multiplication operators, Trans. Amer. Math. Soc. 309 (1988), 711-723.

6. D. Békollé, C. A. Berger, L. A. Coburn, and K. H. Zhu, BMO in the Bergman metric on bounded symmetric domains, J. Funct. Anal. 93 (1990), 310-350.

7. C. A. Berger and L. A. Coburn, Toeplitz operators on the Segal-Bargmann space, Trans. Amer. Math. Soc. 301 (1987), 813-829.

8. C. A. Berger, L. A. Coburn, and K. H. Zhu, Function theory on Cartan domains and the Berezin-Toeplitz symbol calculus, Amer. J. Math. 110 (1988), 921-953.

9. John B. Conway, Subnormal operators, Pitman, London, 1981.

10. J. Faraut and A. Korányi, Function spaces and reproducing kernels on bounded symmetric domains, J. Funct. Anal. 88 (1990), 64-89.

11. P. R. Halmos and V. S. Sunder, Bounded integral operators on $L^{2}$-spaces, Springer, BerlinHeidelberg, 1978.

12. Kenneth Hoffman, Bounded analytic functions and Gleason parts, Ann. of Math. 86 (1967), 74-111.

13. Steven G. Krantz, Function theory of several complex variables, Wiley, New York, 1982.

14. Gerald McDonald, Fredholm properties of a class of Toeplitz operators on the ball, Indiana Univ. Math. J. 26 (1977), 567-576.

15. Gerald McDonald and Carl Sundberg, Toeplitz operators on the disc, Indiana Univ. Math. J. 28 (1979), 595-611.

16. Walter Rudin, Function theory in the unit ball of $\mathbb{C}^{n}$, Springer, Berlin, 1980.

17. Karel Stroethoff, Compact Hankel operators on the Bergman space, Illinois J. Math. 34 (1990), 159-174.

18. __ Compact Hankel operators on the Bergman spaces of the unit ball and the polydisk in $\mathbb{C}^{n}$, J. Operator Theory 23 (1990), 153-170.

19. __ Hankel and Toeplitz operators on the Fock space, Michigan Math. J. (to appear).

20. Dechao Zheng, Hankel operators and Toeplitz operators on the Bergman space, J. Funct. Anal. 83 (1989), 98-120.

21. __ Toeplitz operators and Hankel operators on the Bergman space, Integral Equations Operator Theory 12 (1989), 280-299.

22. __ Semi-commutators of Toeplitz operators on the Bergman space, preprint.

23. Kehe Zhu, VMO, ESV, and Toeplitz operators on the Bergman space, Trans. Amer. Math. Soc. 302 (1987), 617-646.

Department of Mathematical Sciences, University of Montana, Missoula, Montana 59812

Department of Mathematics, State University of New York, Stony Brook, New York 11794 